\title{
Large-eddy simulation of a mildly curved open-channel flow
}

\author{
W. VAN BALEN ${ }^{1} \uparrow$, W. S. J. UIJTTEWAAL $L^{1}$ \\ AND K. B LAN CKAER T T $^{1,2}$ \\ ${ }^{1}$ Department of Civil Engineering and Geosciences, Delft University of Technology, Delft, PO Box 5048, \\ The Netherlands \\ ${ }^{2}$ ICARE-ENAC, Ecole Polytechnique Fédérale, Lausanne, CH-1015, Switzerland
}

(Received 16 October 2008 and in revised form 24 March 2009)

After validation with experimental data, large-eddy simulation (LES) is used to study in detail the open-channel flow through a curved flume. Based on the LES results, the present paper addresses four issues. Firstly, features of the complex bicellular pattern of the secondary flow, occurring in curved open-channel flows, and its origin are investigated. Secondly, the turbulence characteristics of the flow are studied in detail, incorporating the anisotropy of the turbulence stresses, as well as the distribution of the kinetic energy and the turbulent kinetic energy. Moreover, the implications of the pattern of the production of turbulent kinetic energy is discussed within this context. Thirdly, the distribution of the wall shear stresses at the bottom and sidewalls is computed. Fourthly, the effects of changes in the subgrid-scale model and the boundary conditions are investigated. It turns out that the counter-rotating secondary flow cell near the outer bank is a result of the complex interaction between the spatial distribution of turbulence stresses and centrifugal effects. Moreover, it is found that this outer bank cell forms a region of a local increase of turbulent kinetic energy and of its production. Furthermore, it is shown that the bed shear stresses are amplified in the bend. The distribution of the wall shear stresses is deformed throughout the bend due to curvature. Finally, it is shown that changes in the subgrid-scale model, as well as changes in the boundary conditions, have no strong effect on the results.

\section{Introduction}

\subsection{Background}

In open-channel bends, the curvature of the flow gives rise to secondary flow, resulting in the classical helical motion. This helical motion is of large importance in river bends (see, for instance, Einstein \& Harder 1954 and Tanner 1963), where it plays a key role in the formation of erosion and sedimentation patterns in the river bed.

To enable the prediction of the morphological changes in river bends, the flow and turbulence properties have to be known first, since the topography and the planimetry of a river bed strongly depend on the spatial distribution of the velocities and the boundary shear stresses, which directly follow from the Reynolds shear stress distribution. Moreover, the proper understanding of the flow and turbulence 


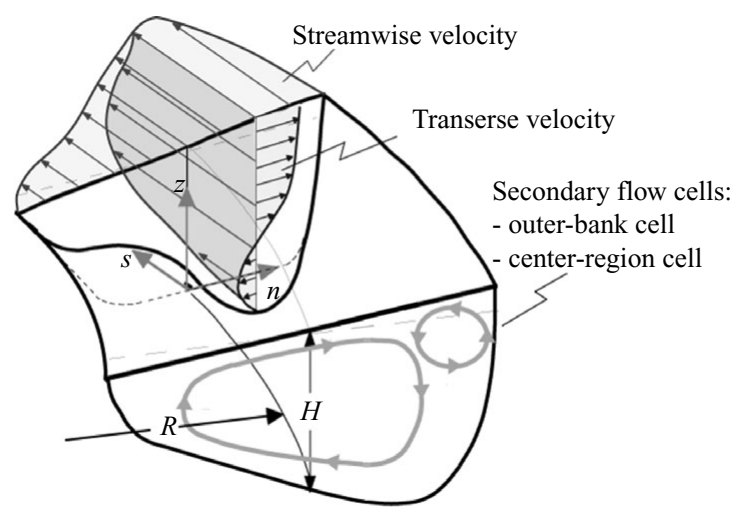

FIGURE 1. Definition sketch of curved open-channel flow.

characteristics in curved open-channel flows is of importance for the prediction of the spreading of pollutants and thus for the water quality of natural river systems.

However, due to the occurring high Reynolds number, the complexity of the river bed and the shallowness of the flow, it is difficult to predict the flow structure in river bends with sufficient detail. In order to understand its features, river flow is often downscaled to a laboratory scale either as a single-bend flow (see, for instance, Rozovskii 1957; Hicks, Jin \& Steffler 1990; Booij 2003) or as a periodic meandering flow (see, for instance, Shiono \& Muto 1998). Field measurements of natural river bend flow are performed by Bathurst, Thorne \& Hey (1977) and Thorne et al. (1985).

One of the key features of curved open-channel flow is the existence of secondary flow cells perpendicular to the main flow direction. In general, two types of secondary flow are distinguished, namely secondary flow of Prandtl's first kind and Prandtl's second kind (Bradshaw 1987; Nezu \& Nakagawa 1993). The first type is associated with vorticity induced by skewing of the mean shear, for instance by centripetal forces. The second type is associated with vorticity generated by anisotropy and inhomogeneity of the Reynolds stress tensor and is commonly referred to as turbulence-driven secondary flow.

In turbulent straight open-channel flow, these turbulence-driven secondary currents have received a lot of attention in the past century, starting with the ideas of Prandtl in the mid twenties. The discussion on these secondary currents seems to be settled in an article by Demuren \& Rodi (1984), who have summarized and criticised the experimental findings of Brundett \& Baines (1964), Gessner \& Jones (1965), Perkins (1970) and Gessner (1973), and have shown, on the basis of the balance equation for streamwise vorticity, that the terms associated with turbulence stresses are dominant, confirming the turbulence stress distribution to be the cause of the secondary flow.

In turbulent curved open-channel flow, secondary flow of Prandtl's first kind comes into existence due to the balance between pressure forces and centripetal forces. Addition of this secondary flow to the primary flow leads to the classical helical motion, which is always observed, for instance, in river bends. Besides the skewinduced secondary flow cell, a second, counter-rotating secondary flow cell often exists near the outer bank of the channel. Figure 1 illustrates this secondary flow pattern schematically. This outer bank cell has been observed in natural river systems by Bathurst et al. (1977) and Thorne et al. (1985). In spite of several attempts to investigate the characteristic features of this outer bank cell, for instance by Booij (2003) and Blanckaert \& de Vriend (2004), it is not yet clear what causes the existence of this outer bank cell. 
Secondary flow in open-channel flow has implications for its numerical modelling. For instance, Demuren \& Rodi (1984) have already pointed out that secondary flow of Prandtl's second kind in straight open-channel flow could not be reproduced by Reynolds Averaged Navier-Stokes (RANS) models with an isotropic eddy viscosity model, as it is the anisotropy of the turbulence stresses that make the secondary flow come into existence (cf. Speziale 1987). Moreover, Blanckaert \& de Vriend (2004) have shown that such models are inherently unable to reproduce the cross-sectional flow pattern of turbulent kinetic energy fluxes as observed by them, because isotropic eddy viscosity models are absolutely dissipative. Thus, the two-way transfer of turbulent kinetic energy between mean motion and turbulent motion cannot be maintained. This is confirmed by the simulations of Kimura et al. (2008) and Zeng et al. (2008).

\subsection{Methodology and objectives}

In the present study, large-eddy simulation (LES) is used for the simulation of the flow through a curved laboratory flume, which can be regarded as a schematisation of the flow through a natural river bend. Since in an LES the flow and turbulence are for the larger part resolved, it enables a thorough study of the large-scale turbulence characteristics of curved open-channel flow, which can largely benefit the understanding of the physical phenomena occurring in natural river bend flow.

LES has become quite a common tool for the study of open-channel flows. Within the context of straight open-channel flow, the work of, among others, Shi, Thomas \& Williams (2000) and Broglia, Pascarelli \& Piomelli (2003) (free-surface effects in open-channel flow), Koken \& Constantinescu (2008) (open-channel flow around spur dikes), McCoy, Constantinescu \& Weber (2008) (open-channel flow with groynes) and Calhoun \& Street (2001) (open-channel flow over a wavy bed) can be mentioned. Recently, Stoesser, Ruether \& Olsen (2008) have carried out an LES of a meandering open-channel flow with a special focus on the near-bed behaviour of the flow.

Nonetheless, on single-bend open-channel flow only RANS studies have been published in the literature. Some of them are reported by, among others, Ye \& McCorquodale (1998) and Zeng et al. (2008). But since the single-bend open-channel flow is a very basic flow type and the most straightforward way to schematise a river bend flow, its flow and turbulence properties have to be known well. For that purpose, RANS studies are not sufficient and a clear need exists for more fundamental numerical studies.

Experimental studies on single-bend open-channel flow are amply found in the literature, starting with Rozovskii (1957) up to the work of Tominaga, Nagao \& Nezu (1999), Blanckaert \& Graf (2001), Booij (2003) and Blanckaert \& de Vriend (2004). However, these studies do not always reveal the turbulence structure of the flow in sufficient detail and/or are restricted to only one cross-section. Moreover, it is very difficult to accurately measure the distribution of the occurring wall shear stresses.

In order to fulfil the need for detailed information and profound knowledge, the main focus of the present LES study is on the flow characteristics and turbulence structure of a single-bend open-channel flow. In this respect, special emphasis is put on the complex features and origin of the secondary flow currents, the structure of the turbulence and the magnitude and distribution of the wall shear stresses.

The outline of this paper is as follows. In $\S 2$, the used computational technique, as well as the turbulence modelling tools, is described. In $\S 3$, the results of the LES are summarized. After validation with experimental data $(\S 3.1)$, the features and origin of the secondary flow currents are highlighted first ( $\$ 3.2)$. Thereafter, in $\S \S 3.3-3.5$, attention is paid to the anisotropy of the occurring turbulence stresses $(\S 3.3)$, the distribution of the kinetic energy and turbulent kinetic energy $(\S 3.4)$ and 

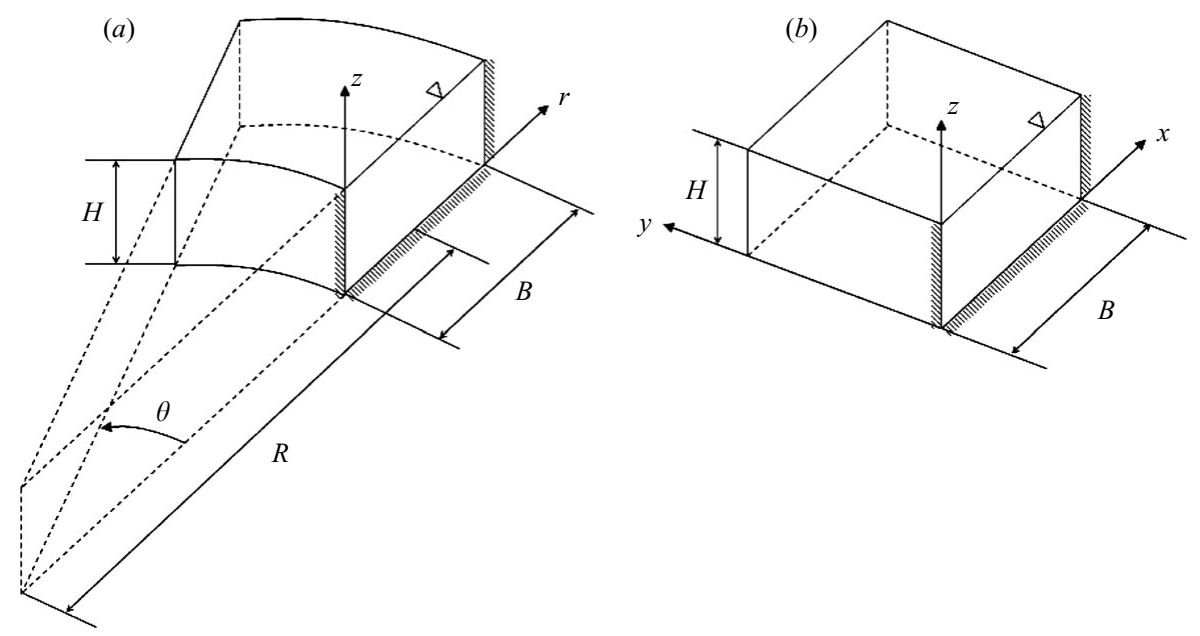

FIGURE 2. (a) Cylindrical coordinates system, with $R$ the radius at the channel axis.

(b) Cartesian coordinates system. $B$ is the width and $H$ is the height of the channel.

the production of turbulent kinetic energy and its consequences for the numerical modelling of curved open-channel flows ( $\$ 3.5)$. In $\S 3.6$, the distribution of the wall shear stresses is discussed, whereafter in $\S 3.7$ the influences of changes in the subgridscale model and the wall model are investigated. The main results and conclusions are summarized in $\S 4$.

\section{Model description}

\subsection{Flow equations}

Within a cylindrical coordinate system with $r, \theta$ and $z$ being the transverse, streamwise and vertical direction (see figure $2 a$ ) with associated velocity components $u, v$ and $w$ respectively, the equations of motion read

$$
\begin{aligned}
\frac{1}{r} \frac{\partial r u}{\partial r}+\frac{1}{r} \frac{\partial v}{\partial \theta}+\frac{\partial w}{\partial z} & =0 \\
\frac{\partial u}{\partial t}+\frac{1}{r} \frac{\partial r u u}{\partial r}+\frac{1}{r} \frac{\partial u v}{\partial \theta}+\frac{\partial u w}{\partial z}-\frac{v^{2}}{r} & =-\frac{\partial p}{\partial r}+v\left(\nabla^{2} u-\frac{u}{r^{2}}-\frac{2}{r^{2}} \frac{\partial v}{\partial \theta}\right), \\
\frac{\partial v}{\partial t}+\frac{1}{r} \frac{\partial r v u}{\partial r}+\frac{1}{r} \frac{\partial v v}{\partial \theta}+\frac{\partial v w}{\partial z}+\frac{u v}{r} & =-\frac{1}{r} \frac{\partial p}{\partial \theta}+v\left(\nabla^{2} v-\frac{v}{r^{2}}+\frac{2}{r^{2}} \frac{\partial u}{\partial \theta}\right), \\
\frac{\partial w}{\partial t}+\frac{1}{r} \frac{\partial r w u}{\partial r}+\frac{1}{r} \frac{\partial w v}{\partial \theta}+\frac{\partial w w}{\partial z} & =-\frac{\partial p}{\partial z}+v \nabla^{2} w
\end{aligned}
$$

where $\nabla^{2}$ is defined as

$$
\nabla^{2} \equiv \frac{1}{r} \frac{\partial}{\partial r}\left(r \frac{\partial}{\partial r}\right)+\frac{1}{r^{2}} \frac{\partial^{2}}{\partial \theta^{2}}+\frac{\partial^{2}}{\partial z^{2}}
$$

Because the density $\rho$ is taken constant, it is absorbed in the pressure $p$. The equations for the straight parts of the single-bend configuration are obtained from (2.1)-(2.4) by letting $1 / R \rightarrow 0$. The coordinates $r, \theta$ and $z$ then change into $x, y$ and $z$ (see figure $2 b$ ). Notice that in that case $r \partial \theta \rightarrow \partial y$.

The equations are solved on a staggered mesh using the finite-volume method, with typical grid cells as shown in figure 3, using a pressure-correction algorithm. These 


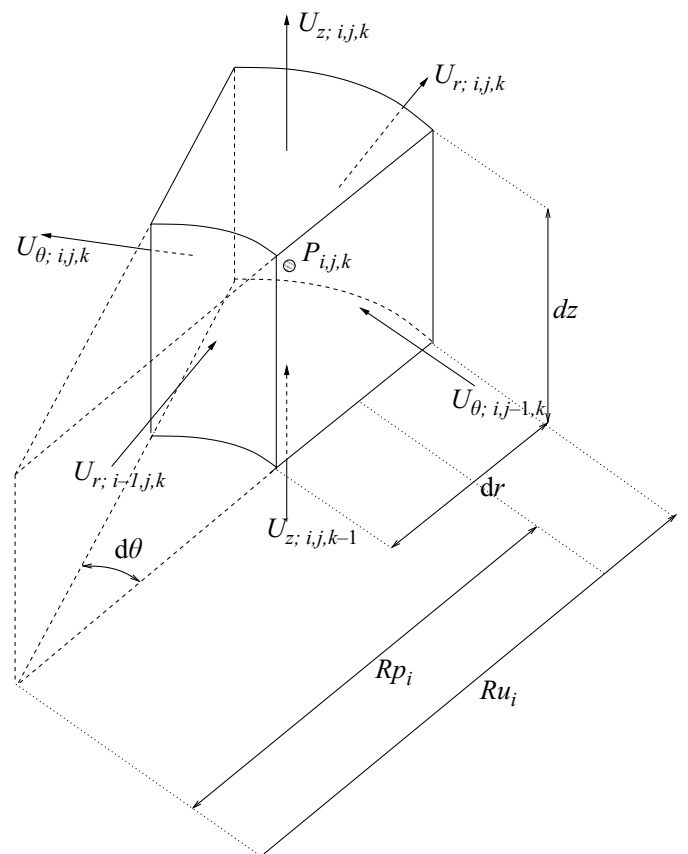

FiguRE 3. Typical grid cell in the cylindrical staggered mesh.

equations are numerically integrated in space using the midpoint rule. As a matter of fact, this procedure results in the spatial discretization of the domain following the second-order central scheme. The equations are integrated in time using the explicit second-order Adams-Bashforth scheme. More details on the numerics can be found in Pourquié (1994).

\subsection{Turbulence modelling}

In the LES model, (2.1)-(2.4) are spatially filtered by a top-hat filter defined by the grid spacing. The subgrid-scale viscosity $v_{s g s}$, needed for the modelling of the subgrid-scale stress tensor arising from the filtering operation, is modelled using Smagorinsky's model:

$$
v_{s g s}=C_{s}^{2} \Delta^{2}\left|\widetilde{S}_{i j}\right|,
$$

where $C_{s}$ is Smagorinsky's constant and $\Delta$ is the filterlength, defined as $\Delta=(r \Delta r \Delta \theta \Delta z)^{1 / 3}$, and $\widetilde{S}_{i j}$ is the rate of strain tensor based on the filtered velocities. In this paper, the value for Smagorinsky's constant is taken $C_{s}=0.1$. A standard Van Driest damping function is used in order to let $v_{s g s} \rightarrow 0$ at solid walls.

An alternative to this standard Smagorinsky model is the dynamic Smagorinsky model, as proposed by Germano et al. (1991) in the modified version of Lilly (1992). This subgrid-scale model replaces the constant $C_{s}^{2}$ in (2.6) by $C_{d}$, so that

$$
v_{s g s}=C_{d} \Delta^{2}\left|\widetilde{S}_{i j}\right| \text {. }
$$

The model constant $C_{d}$ is calculated via

$$
C_{d}=\frac{L_{i j} M_{i j}}{M_{i j} M_{i j}},
$$




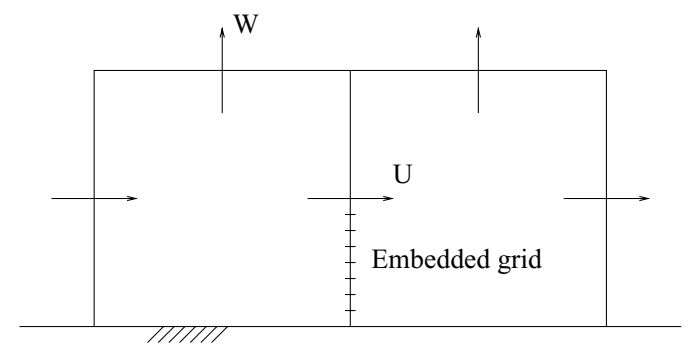

FIgURE 4. Two grid cells adjacent to the wall. The arrows denote velocity points in the computational staggered grid. The small lines between the solid wall and the most near-wall velocity point denote the embedded second grid on which the simplified boundary-layer equation is solved.

where $L_{i j}$ and $M_{i j}$ are defined as

$$
L_{i j}=\widehat{\widetilde{u}}_{i} \widetilde{\widetilde{u}}_{j}-\widehat{\widetilde{u}}_{i} \widehat{\widetilde{u}}_{j} \quad \text { and } \quad M_{i j}=2 \Delta^{2}\left|{\widehat{\widetilde{S}} \mid \widetilde{S}_{i j}}-2 \widehat{\Delta}^{2}\right| \widehat{\widetilde{S}} \widehat{\widetilde{S}}_{i j}
$$

In these equations, the hat $\widehat{\cdot}$ denotes spatial filtering over the testfilter, which is defined as the spatial average of the values at a certain cell and its $3^{3}-1$ neighbours. In order to avoid instabilities due to a negative viscosity, it is required that $v+v_{s g s} \geqslant 0$. In this paper, either the standard Smagorinsky model or the dynamic Smagorinsky model is used.

Reynolds decomposition of the resolved field is denoted as $\widetilde{u}_{i}=\widetilde{U}_{i}+\widetilde{u}_{i}^{\prime}$. In the following, the tilde $\sim$ will be omitted.

\subsection{Boundary conditions}

In our simulations of curved open-channel flow, the free surface is treated as a horizontal rigid lid where free-slip conditions are applied. The assumption of a freestress rigid lid is justified as the longitudinal and transverse water-level slopes are negligibly small. The rigid lid assumption is often used for the representation of the free surface of an open-channel flow (see, for instance, Pan \& Banerjee 1995 and Broglia et al. 2003). A convective boundary condition is used at the outflow.

Hydraulically smooth solid walls are represented by the two-layer model as proposed by Balaras, Benocci \& Piomelli (1996). This model solves $u_{i}$ on an embedded second grid which is located between the solid wall and the most near-wall velocity point of the computational staggered grid (see figure 4), following

$$
\frac{\partial}{\partial x_{n}}\left(\left(v+v_{t}\right) \frac{\partial u_{i}}{\partial x_{n}}\right)=F_{i},
$$

with

$$
F_{i}=\frac{\partial u_{i}}{\partial t}+\frac{\partial u_{i} u_{j}}{\partial x_{j}}+\frac{\partial p}{\partial x_{i}},
$$

and $n$ the direction normal to the wall and $i, j$ the directions parallel to the wall. The basic rationale behind this method is to solve a simplified one-dimensional boundarylayer equation between the solid wall and the velocity point closest to the solid wall rather than to refine the whole computational grid towards the wall and to resolve the whole boundary layer which is obviously computationally more expensive. 
For the eddy viscosity $v_{t}$ on this so-called embedded grid, a Prandtl mixing length model is adopted, which includes Van Driest damping (cf. Tessicini et al. 2002):

$$
\frac{v_{t}}{v}=\kappa x_{n}^{+}\left(1-\exp \left(-\frac{x_{n}^{+}}{A}\right)\right)^{2},
$$

with $\kappa=0.4$ and $A=19$. It can be shown that if $F_{i}=0$, the solution of $(2.10)$ is the standard law of the wall, including the viscous sublayer, the buffer layer and the logarithmic layer.

\subsection{The pressure Poisson equation}

Since the standard pressure-correction method is used in order to solve the flow equations, the pressure Poisson equation has to be solved. In the cylindrical coordinate system, this equation reads

$$
\frac{1}{r} \frac{\partial}{\partial r}\left(r \frac{\partial p}{\partial r}\right)+\frac{1}{r^{2}} \frac{\partial^{2} p}{\partial \theta^{2}}+\frac{\partial^{2} p}{\partial z^{2}}=\text { right-handside },
$$

where right-hand side involves the divergence of the predicted velocity field.

For homogeneous meshes, fast direct solvers based on Fast Fourier Transformations (FFT) are readily available (www.netlib.org). For the single-bend case studied in this paper, the FFT can be used only in the vertical direction $z$, since the radial term in (2.13) cannot be directly transformed into Fourier space and the tangential grid size $r \Delta \theta$ is not homogeneous anymore. If the FFT is used in the $z$-direction with $N_{z}$ grid points, then the resulting $N_{z}$ decoupled Helmholtz equations cannot be solved using a fast direct solver. Therefore, the iterative method BiCGSTAB of van der Vorst (1992) in combination with the modified ILU preconditioner of Gustafsson (1978) is adopted. A serious disadvantage of this method is that it is much slower than a direct solver using an FFT in two directions.

However, we can show that for our particular single-bend geometry, an important simplification can be made, which enables the use of an FFT in two directions and a double-sweep algorithm in the remaining third direction. Therefore, we consider the non-conservative equivalent of (2.13):

$$
\frac{\partial^{2} p}{\partial r^{2}}+\frac{1}{r} \frac{\partial p}{\partial r}+\frac{1}{r^{2}} \frac{\partial^{2} p}{\partial \theta^{2}}+\frac{\partial^{2} p}{\partial z^{2}}=\text { right-handside. }
$$

We now focus on the discretized version of the two terms with derivatives in the $r$-direction, which is given by

$$
\begin{aligned}
\frac{\partial^{2} p}{\partial r^{2}}+\frac{1}{r} \frac{\partial p}{\partial r} & =\frac{p_{i+1}-2 p_{i}+p_{i-1}}{\Delta r^{2}}+\frac{1}{r_{i}} \frac{p_{i+1}-p_{i-1}}{2 \Delta r} \\
& =\left(\frac{1}{\Delta r^{2}}+\frac{1}{2 r_{i}} \frac{1}{\Delta r}\right) p_{i+1}+\left(\frac{-2}{\Delta r^{2}}\right) p_{i}+\left(\frac{1}{\Delta r^{2}}-\frac{1}{2 r_{i}} \frac{1}{\Delta r}\right) p_{i-1} .
\end{aligned}
$$

In our simulations, we take $\Delta r$ constant. It is seen that the off-diagonal terms (i.e. the $p_{i+1}$ term and the $p_{i-1}$ term) consist of two parts, namely a part arising from the discretization of the second derivative and a part arising from the discretization of the first derivative. The ratio of their contributions to the discretized system is

$$
\frac{\frac{1}{\Delta r^{2}}}{\frac{1}{2 r_{i} \Delta r}}=\frac{2 r_{i}}{\Delta r}=\frac{2 r_{i} N_{r}}{B}
$$




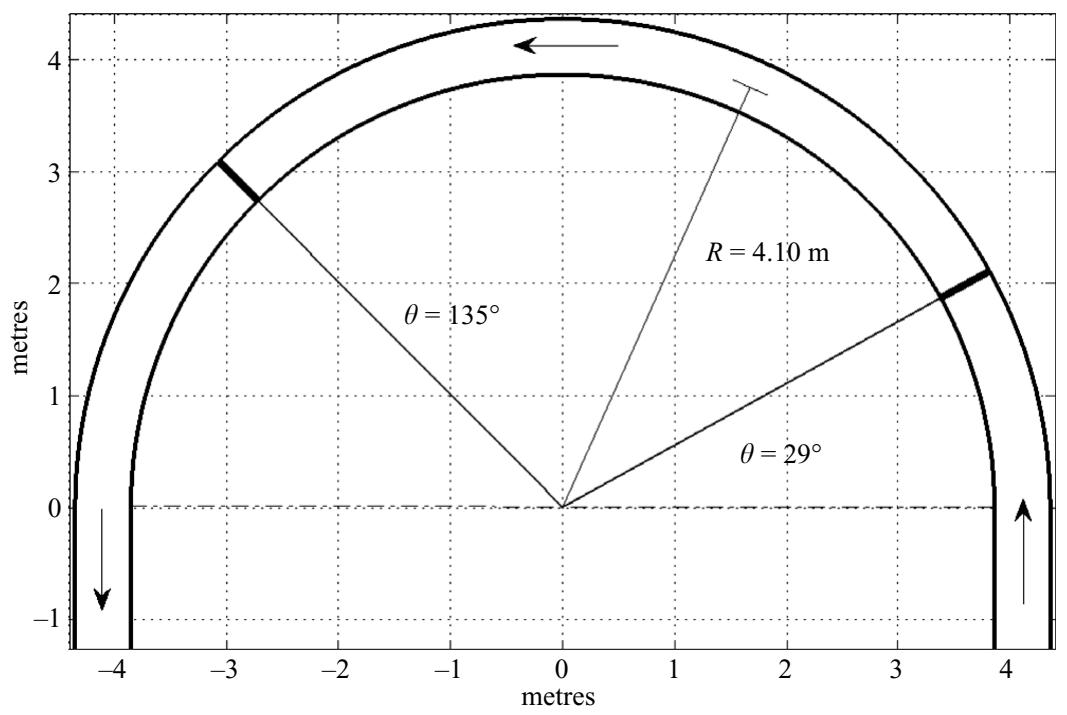

FIgURE 5. Computational domain for the entire curved flume.

with $B$ the width of the channel and $N_{r}$ the number of grid cells in $r$-direction. The actual values in this paper are $r=4.1 \mathrm{~m}, B=0.5 \mathrm{~m}$ and $N_{r}=168$. If these typical values are substituted in (2.17), it is seen that the ratio of the two contributions to the off-diagonal coefficients is 2755 , which indicates that the contribution of the second derivative in (2.14) is by far dominant. Hence, we neglect the term $1 / r \cdot \partial p / \partial r$ in our applications. Neglecting this term has the advantage that it enables the use of an FFT in both the $r$-direction and the $z$-direction.

Since this assumption substantially accelerates the pressure solver, the FFT-based method is chosen for our particular purpose rather than the BiCGSTAB-based method. In Appendix A it is shown that this choice is justified for our particular problem. However, for very sharp bends in which case the term $1 / r \cdot \partial p / \partial r$ is not negligible anymore, the BiCGSTAB-based method must be used. But this is not the case for the geometry that is studied in this paper.

\subsection{Flow geometry}

The physical domain is the U-shaped flume as used by Booij (2003) (shown in figure 5). This experimental flume consists of a straight inflow part of 11 metres, a curved part of $180^{\circ}$, and a straight outflow part of 6.7 metres. The solid sidewalls and the solid bottom are hydraulically smooth. The hydraulic conditions for the simulations are given in table 1 . With a radius-to-depth ratio of $R / H=79$ and a radius-to-width ratio of $R / B=8$, the curvature of the flume can be qualified as relatively mild. In the experiments by Booij (2003), it was observed that tangential velocity gradients are negligible beyond approximately $90^{\circ}$ in the bend. Hence, the flow in the far field of the bend can be considered as axisymmetric.

Five simulations are performed of the present flow case (see table 2). These five simulations can be divided into two types of simulations. The first type (Run 1) is the simulation of the spatially developing flow through the entire flume as given in figure 5. The second type (Runs 2-5) is the simulation of the axisymmetric part of the bend flow, as it is observed in the far field of the single bend. 


$\begin{array}{cccccccccc}R(\mathrm{~m}) & B(\mathrm{~m}) & H(\mathrm{~m}) & Q\left(1 \mathrm{~s}^{-1}\right) & V_{a v}\left(\mathrm{~m} \mathrm{~s}^{-1}\right) & R e & F r & R / B & R / H & B / H \\ 4.1 & 0.5 & 0.052 & 5.2 & 0.2 & 10400 & 0.28 & 8.2 & 78.8 & 9.6\end{array}$

TABLE 1 . Hydraulic conditions. $R$ denotes the radius at the centreline, $B$ is the width of the flume, $H$ is the water depth, $Q$ is the discharge, $V_{a v}$ is the bulk velocity, $R e$ is the Reynolds number and $F r$ is the Froude number. $R e$ and $F r$ are based on the bulk velocity $V_{a v}$ and the waterdepth $H$.

$\begin{array}{lcccc} & \text { Subgrid-scale model } & F_{i} & \text { Boundary conditions } & \text { Mesh } \\ \text { Run 1 } & \text { standard Smagorinsky } & 0 & \text { Non-periodic } & 3600 \times 168 \times 24 \\ \text { Run 2 } & \text { standard Smagorinsky } & 0 & \text { Periodic } & 300 \times 168 \times 24 \\ \text { Run 3 } & \text { dynamic Smagorinsky } & \partial p / \partial x_{i} & \text { Periodic } & 300 \times 168 \times 24 \\ \text { Run 4 } & \text { standard Smagorinsky } & 0 & \text { Periodic } & 300 \times 168 \times 24 \\ \text { Run 5 } & \text { dynamic Smagorinsky } & \partial p / \partial x_{i} & \text { Periodic } & 300 \times 168 \times 24\end{array}$

TABle 2. Model settings for the different runs. $F_{i}$ refers to (2.11), the mesh is given as the number of grid cells in streamwise, transverse and vertical direction.

Run 1 is a simulation of the flow through the entire flume. The lengths of both the straight inflow part and the straight outflow part were shortened to $1.27 \mathrm{~m}$ in the computational model. The mesh used for Run 1 consists of $168 \times 3600 \times 24$ grid cells in transverse, streamwise and vertical direction, respectively (14.5 million grid cells in total). The standard Smagorinsky model is used for the subgrid-scale stresses. For the boundary conditions, the value $F_{i}=0$ is used in (2.11). The inflow conditions are provided by a previously performed simulation of a straight open-channel flow at the corresponding Reynolds number. The distance between the wall and the first computational point is typically of the order of $10-15$ wall units. The simulation was given 240 seconds simulated time to reach a statistically steady state, which was found to be amply long enough. The results were time averaged over a period of 900 seconds physical time.

Runs 2-5 are simulations of the axisymmetric flow in the far field of the bend. Since for this purpose periodic boundary conditions can be used in streamwise direction, Runs $2-5$ can be performed at much lower computational costs compared to Run 1. The mesh used for Runs $2-5$ consists of $168 \times 300 \times 24$ grid cells in transverse, streamwise and vertical direction, respectively ( 1.2 million grid cells in total). The mutual differences between Runs 2, 3, 4 and 5 are the choices for the subgrid-scale model and the wall model. In this way, we can investigate to what extent the choices for the subgrid-scale model and the wall model affect the solution of the simulation. Because of the low computational costs, it is preferable to use the axisymmetric flow case for this investigation rather than the spatially developing flow case. The standard Smagorinsky model is used for Runs 2 and 3, while the dynamic Smagorinsky model is used for Runs 4 and 5. For the boundary conditions, the value $F_{i}=0$ is used in (2.10) for the Runs 2 and 4, whereas the pressure gradient $\partial p / \partial x_{i}$ is incorporated in (2.11) for the Runs 3 and 5. These simulations were given 200 seconds simulated time to reach a statistically steady state. The results were time averaged over a period of 600 seconds physical time.

Nonetheless, the main focus of the present paper is Run 1, as it most completely reveals the physics of the studied curved open-channel flow, which is the main aim of this paper. The results for the spatially developing flow case (Run 1) are given in 
$\S \S 3.1-3.6$. The results for the axisymmetric flow case (Runs 2-5) are presented in $\S 3.7$. In Appendix B, it is shown on the basis of Run 2 that the solution of the simulations is independent of the chosen mesh.

\section{Results and discussion}

It was seen in the output of the simulation that there is a region of spatial development of the flow (from $0^{\circ}$ to about $70^{\circ}$ ) and a region where the flow can be considered as axisymmetric (beyond about $90^{\circ}$ ). Moreover, it was concluded that the curvature of the flow is so mild that no pronounced flow structures, other than the two secondary flow cells, relevant for our particular purpose are observed that could have evolved due to curvature. In view of our aims it suffices to restrict our analysis for the major part to a cross-section in the near field and in the far field of the flow. Therefore, we consider the $29^{\circ}$ cross-section and the $135^{\circ}$ cross-section. The results at the $135^{\circ}$ cross-section are compared with the experimental data of Booij (2003) and those at the $29^{\circ}$ cross-section with experimental data obtained by Blokland (1985).

\subsection{Model validation}

In figures 6 and 7, some vertical profiles of the time-averaged streamwise, transverse and vertical velocities ( $V, U$ and $W$ respectively), as well as the Reynolds stress components, are shown for the $29^{\circ}$ and $135^{\circ}$ cross-sections. At the $29^{\circ}$ cross-section, the vertical profiles are obtained at 11 locations, which are equidistantly placed across the width. At the $135^{\circ}$ cross-section, nine locations were used in the experiment, also equidistantly distributed across the width. The data are non-dimensionalized using the bulk velocity $V_{a v}=0.2 \mathrm{~m} \mathrm{~s}^{-1}$.

From figures 6 and 7, it can be seen that the agreement of the numerical data with the experimental data is good. It is seen that the velocities of the secondary flow in the core region of the cross-section are generally about $10 \%$ of the bulk velocity. The negative values of the transverse velocities near the outer bank at both cross-sections indicate that the counter-rotating secondary flow cell near the outer bank is present with transverse velocities of the same order of magnitude as of the primary helical motion. The disagreement of the transverse velocities at the outer bank seems to indicate that the size of the outer bank cell at the $29^{\circ}$ cross-section is slightly smaller in the simulation compared to the experiment. The disagreement in the vertical velocities near the outer bank can be explained as suggested by Booij (2003): "The LDV beam configuration used for measuring through the bottom yields the largest error in the already small measured value of the vertical velocity component $W$. Hence the reliability of the obtained $W$ is relatively poor."

Also the Reynolds stresses agree well with the experimental data. The good agreement of the Reynolds stresses is of large importance, for they give the proper wall shear stresses and appear in the balance equation for the streamwise vorticity, which describes the characteristics of the secondary flow currents. It is seen that the $\overline{v^{\prime} w^{\prime}}$ stresses are deformed compared to the pattern that would be expected for straight flow, for which the $\overline{v^{\prime} w^{\prime}}$ profile linearly varies over the depth from zero at the free surface to the friction velocity squared at the bottom. Especially for the $135^{\circ}$ cross-section, it is seen that the profile of the $\overline{v^{\prime} w^{\prime}}$ stresses is only approximately linear in the lower half of the water column.

\subsection{Secondary flow}

In figure 8 , the bicellular pattern of the secondary flow at the $135^{\circ}$ cross-section, as seen in the experiment, is visualized by time-averaged velocity vectors. Notice 
(a) Streamwise velocities $-29.0^{\circ}$ cross-section

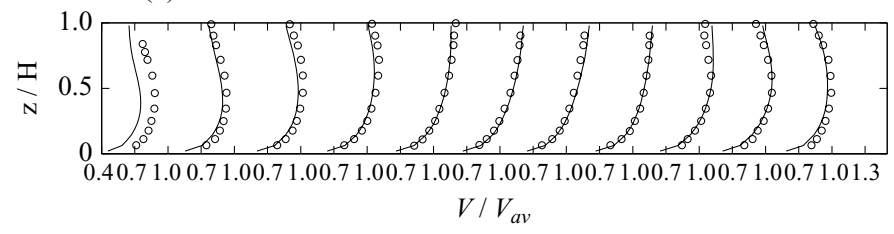

(b) Transverse velocities $-29.0^{\circ}$ cross-section

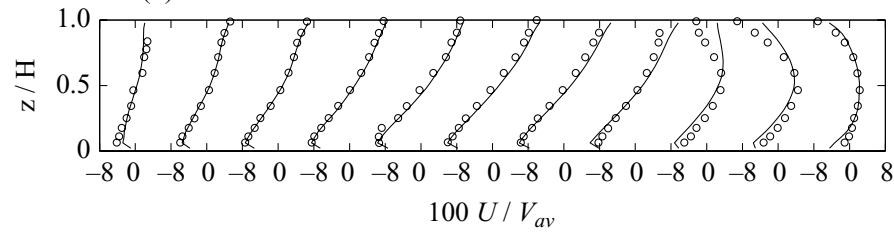

(c) Vertical velocities $-29.0^{\circ}$ cross-section

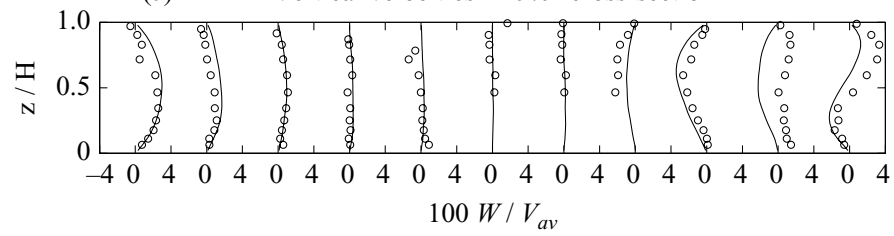

(d) Reynolds stresses v'w' $-29.0^{\circ}$ cross-section

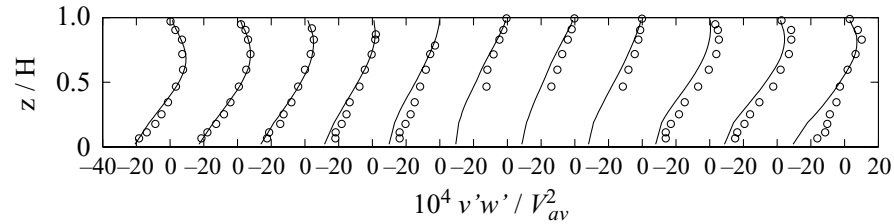

(e) Reynolds stresses u'v' $-29.0^{\circ}$ cross-section
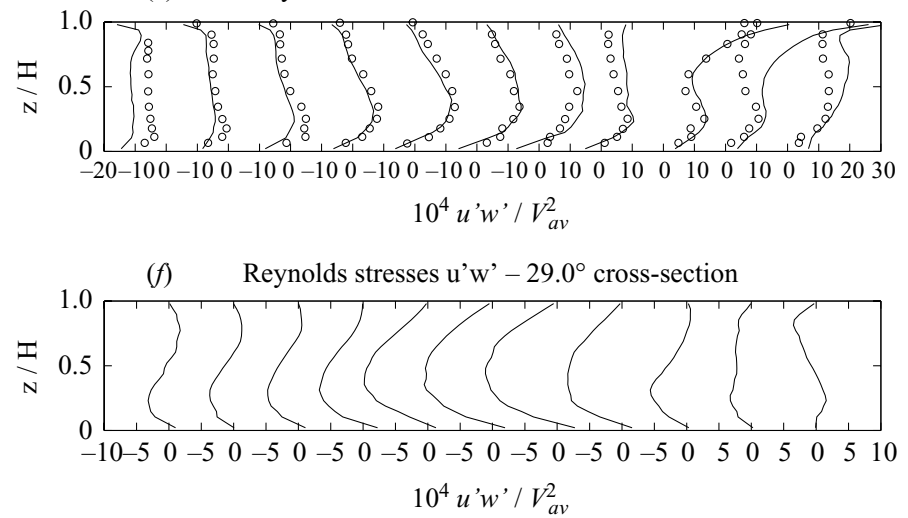

FIGURE 6. Velocity components $(a-c)$ and Reynolds stresses $(d-f)$ from the experiments $(\bigcirc)$ and the LES (-), at locations equidistantly placed from the inner bank (left) to the outer bank (right) for the $29^{\circ}$ cross-section. The values are made non-dimensional using the bulk velocity $V_{a v}=0.2 \mathrm{~m} \mathrm{~s}^{-1}$.

that only the outer $20 \%$ of the width is shown. On the left, a part of the primary circulation cell is seen, whereas at the outer bank a counter-rotating circulation cell is seen. From this figure, the sizes of this outer bank cell can be roughly estimated as $1.2 \mathrm{H} \times 0.5 \mathrm{H}$. Information on the dimensions of the outer bank cell at the $29^{\circ}$ cross-section is not directly available from the experiment. 
(a) Streamwise velocities $-135.0^{\circ}$ cross-section

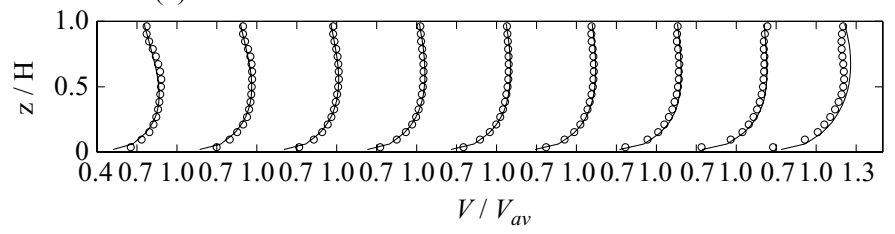

(b) Transverse velocities $-135.0^{\circ}$ cross-section

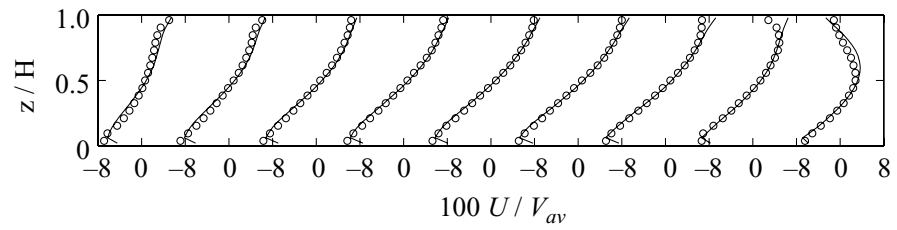

(c) Vertical velocities $-135.0^{\circ}$ cross-section

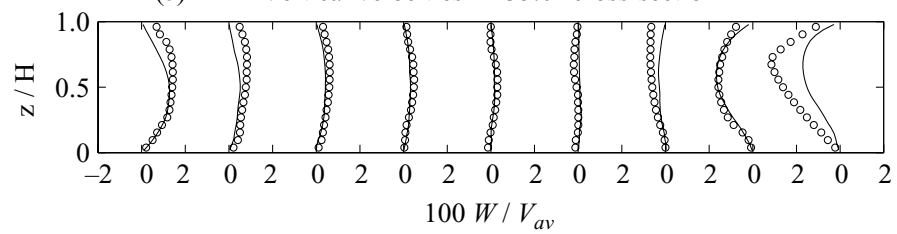

(d) Reynolds stresses v'w' $-135.0^{\circ}$ cross-section

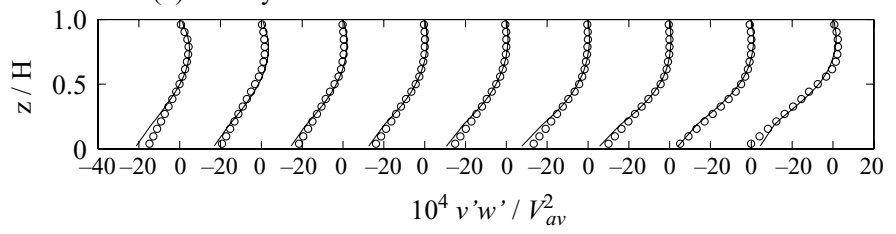

(e) Reynolds stresses u'v' $-135.0^{\circ}$ cross-section

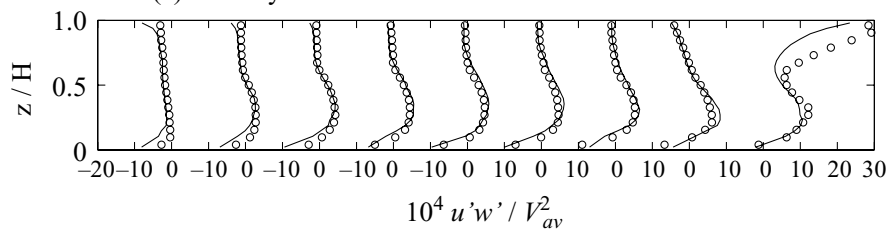

(f) Reynolds stresses u'w' $-135.0^{\circ}$ cross-section

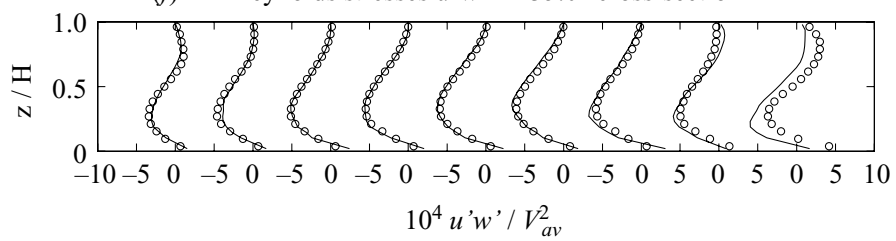

FIGURE 7. Velocity components $(a-c)$ and Reynolds stresses $(d-f)$ from the experiments $(\bigcirc)$ and the LES (-), at locations equidistantly placed from the inner bank (left) to the outer bank (right) for the $135^{\circ}$ cross-section. The values are made non-dimensional using the bulk velocity $V_{a v}=0.2 \mathrm{~m} \mathrm{~s}^{-1}$. 


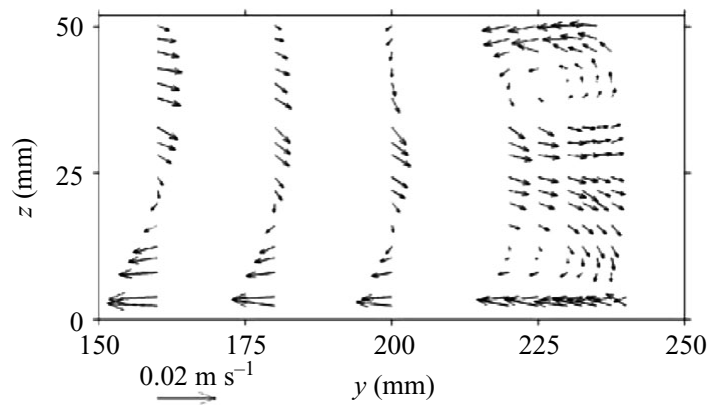

FIGURE 8. Secondary flow pattern, measured by Booij (2003) in the region near the outer bank at the $135^{\circ}$ cross-section in the experiment, visualized by velocity vectors. Only the outer $20 \%$ of the width is shown, from $0.8 B$ to $1.0 B$, with $B=0.5 \mathrm{~m}$ the width of the channel.
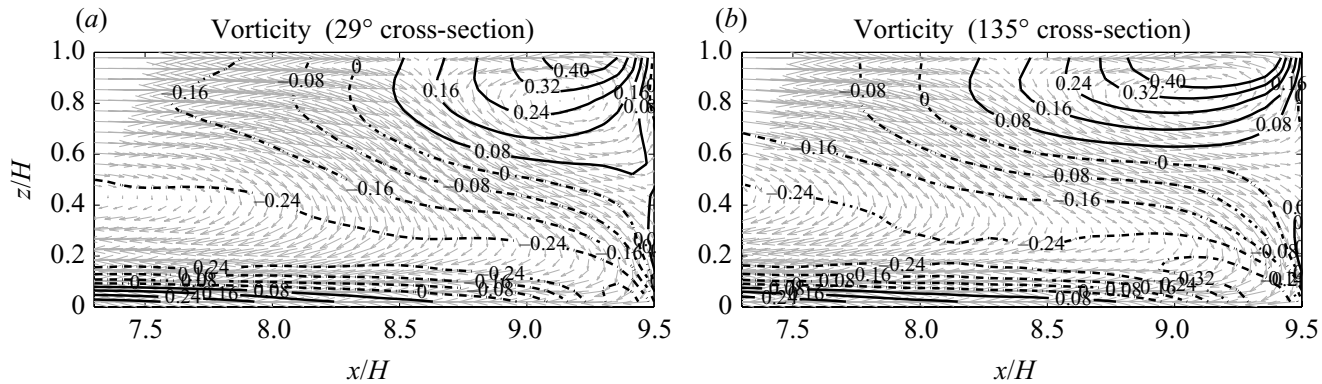

(c) Stream function $\times 10^{3}\left(29^{\circ}\right.$ cross-section $)$

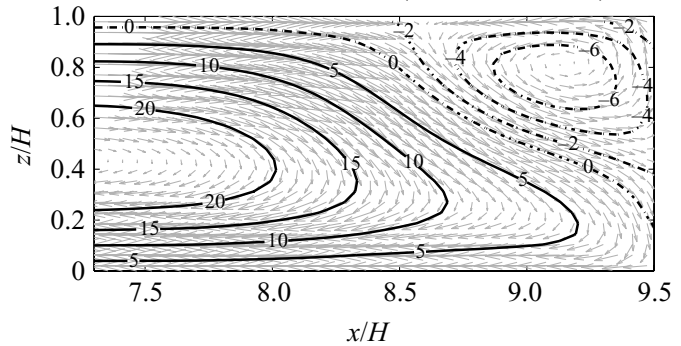

(d) Stream function $\times 10^{3}\left(135^{\circ}\right.$ cross-section $)$

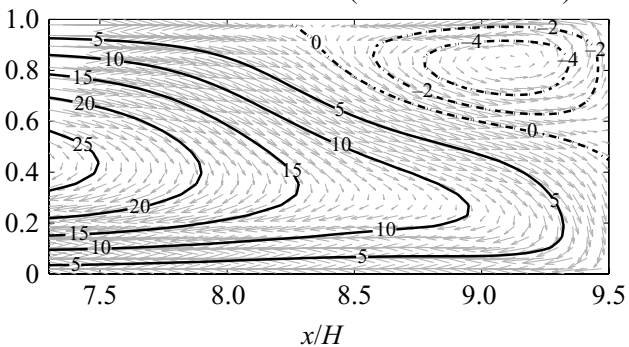

FIGURE 9. Vorticity $\omega_{\theta} H / V_{a v}(a, b)$ and streamfunction $\psi \times 10^{3} / H R V_{a v}(c, d)$ of the flow at the outer bank region of the $29^{\circ}$ cross-section $(a, c)$ and the $135^{\circ}$ cross-section $(b, d)$. Notice that only the outer $23 \%$ of the cross-section is shown. Recall that $x$ denotes the distance from the inner bank.

In figure 9 , the computed streamwise vorticity, defined by

$$
\omega_{\theta}=\frac{\partial W}{\partial r}-\frac{\partial U}{\partial z}
$$

and the computed pseudo-streamfunction $\psi$, related to the streamwise vorticity by $\nabla^{2} \psi=\omega_{\theta}$, are shown for the outer bank area of both the $29^{\circ}$ cross-section and the $135^{\circ}$ cross-section. The vorticity is non-dimensionalized following $\omega_{\theta} H / V_{a v}$ and the streamfunction according to $\psi / H R V_{a v}$. Notice that only the part of the cross-section near the outer bank is shown. In the background of each picture, the velocity vectors are shown. Figure 9 clearly shows the bicellular pattern of the secondary flow. 
A comparison of figure $9(d)$ with figure 8 shows that the dimensions of the outer bank cell at the $135^{\circ}$ cross-section, as seen in the numerical results, agree quite well with those as observed in the experiment, since the sizes of the outer bank cell can be roughly estimated as $1.2 H \times 0.5 H$ both in the LES results and the experimental results. It was found that there is not much variation in the sizes of the outer bank cell along the flume. The strength of the outer bank cell, expressed in terms of $10^{3} \times \psi / H R V_{a v}$, develops from -3 at the inlet of the bend, via a maximum of -8 at the $25^{\circ}$ cross-section, to an equilibrium value of -6 in the far field of the bend. The centre region cell gains strength throughout the flume with values of about 25 in the near field up to 30 in the far field.

We try to gain insight into the origin of the outer bank cell from the equation for streamwise vorticity in cylindrical coordinates. In this equation, we neglect gradients in the tangential direction $\theta$, for it was found that these gradients are small and that they hardly influence the results of the analysis. The equation for the streamwise vorticity $\omega_{\theta}$, defined by $(3.1)$, reads

$$
\frac{\partial \omega_{\theta}}{\partial t}=A D V+C F G+H O M+I S O+D I F F,
$$

where the terms on the right-hand side represent

$$
\begin{aligned}
A D V & =-\left(U \frac{\partial \omega_{\theta}}{\partial r}+W \frac{\partial \omega_{\theta}}{\partial z}\right)+\frac{U}{r} \omega_{\theta}, \\
C F G & =-\frac{1}{r} \frac{\partial}{\partial z}\left(V^{2}+\overline{v^{\prime 2}}\right), \\
I S O & =\frac{\partial^{2}}{\partial r \partial z}\left(\overline{u^{\prime 2}}-\overline{w^{\prime 2}}\right)+\frac{1}{r} \frac{\partial \overline{u^{\prime 2}}}{\partial z}, \\
H O M & =\left(\frac{\partial^{2}}{\partial z^{2}}-\frac{\partial^{2}}{\partial r^{2}}-\frac{1}{r} \frac{\partial}{\partial r}+\frac{1}{r^{2}}\right) \overline{u^{\prime} w^{\prime}},
\end{aligned}
$$

and DIFF the diffusion term, which is left out of consideration. The overlining denotes that averaged quantities are concerned. Furthermore, we define a term $T U R B$ as the sum of $I S O$ and $H O M$, accounting for the contribution of gradients of all the turbulence stresses. In (3.2), $A D V$ represents advection of streamwise vorticity, $C F G$ represents effects that can be associated with centripetal forces, $T U R B$ accounts for effects that can be directly related to turbulence stresses, incorporating anisotropy (the $I S O$-term) and inhomogeneity (the $H O M$-term) of the Reynolds stress tensor. Contour plots of the quantities $A D V, C F G$ and $T U R B$ are given in figure 10 for the $29^{\circ}$ cross-section and the $135^{\circ}$ cross-section. Recall that the rigid lid assumption is used for the free surface. Despite the fact that pressure cannot generate vorticity, the pattern of the vorticity may be affected indirectly by a slightly changed velocity field due to the deformation of the free surface. Currently, we assume that this influence is negligibly small and leave this issue for further investigation.

From figure $10(c, d)$, it becomes clear that the centrifugal term $C F G$ plays a crucial role in the existence of the outer bank cell, as a clear correlation is seen between vorticity and streamfunction on the one hand, and the $C F G$-term on the other hand. The fact that the sign of the $C F G$-term corresponds with the sign of the streamwise vorticity $\omega_{\theta}$ indicates that there is a positive interaction between these two terms. Apparently, the centrifugal effects favour the rotational sense of the secondary flow.

Comparing the results for the two cross-sections (figure 10), it can be seen that the maximum value of the $C F G$-term in the outer bank cell region is higher at the 
(a)

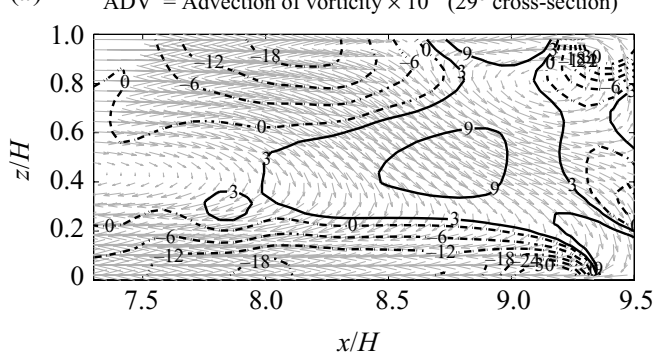

(c) $\quad \mathrm{CFG}=$ Centrifugal term $\times 10^{3}\left(29^{\circ}\right.$ cross-section $)$

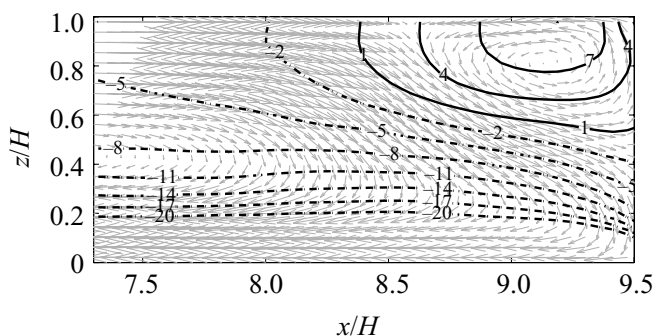

(e) $\mathrm{ISO}+\mathrm{HOM}=$ Turbulence source term $\times 10^{3}\left(29^{\circ}\right.$ cross-section $)$

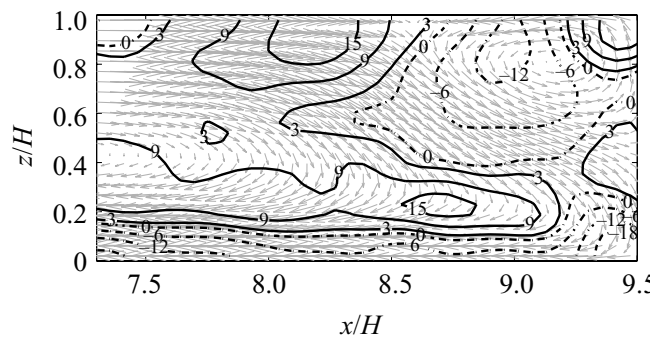

(b) $\mathrm{ADV}=$ Advection of vorticity $\times 10^{3}\left(135^{\circ}\right.$ cross-section $)$

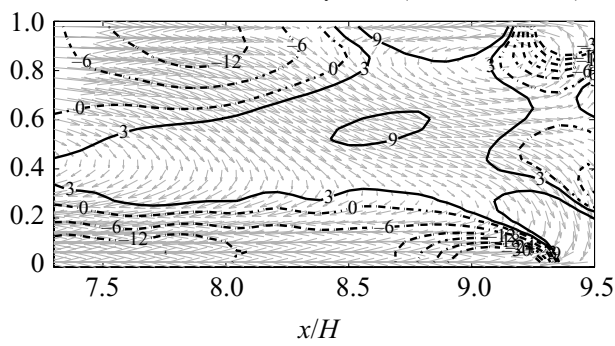

(d) $\quad \mathrm{CFG}=$ Centrifugal term $\times 10^{3}\left(135^{\circ}\right.$ cross-section $)$

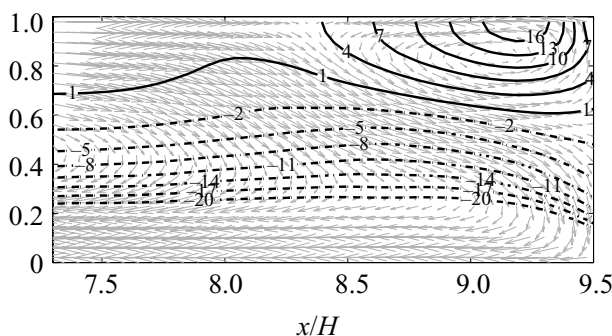

(f) ISO $+\mathrm{HOM}=$ Turbulence source term $\times 10^{3}\left(135^{\circ}\right.$ cross-section $)$

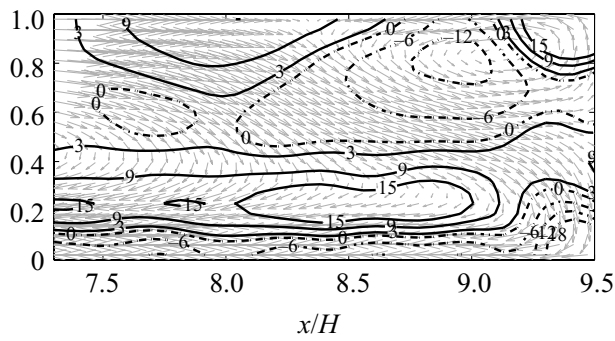

FIGURE 10. Contour plots of the advection term $A D V \times 10^{3} H^{2} / V_{a v}^{2}(a, b)$, the centrifugal term $C F G \times 10^{3} H^{2} / V_{a v}^{2}(c, d)$ and turbulence term $T U R B \times 10^{3} H^{2} / V_{a v}^{2}(e, f)$ for the outer bank region of the $29^{\circ}$ cross-section $\left(a, c\right.$ and $e$ ) and the $135^{\circ}$ cross-section $(b, d$ and $f$ ).

$135^{\circ}$ cross-section compared to the $29^{\circ}$ cross-section. Hence, it can be concluded that farther downstream in the bend, the streamwise velocity profile in the outer bank region is more deformed, since the $C F G$-term is directly related to gradients of the streamwise velocity.

In figure 10 , it is seen that the $A D V$-term and the $T U R B$-term are of opposite sign for the larger part of the outer bank region and thus seem to be opposed to each other. The two terms have in common that a change of sign occurs at locations at the outer bank and the free surface, where the streamfunction $\psi$ changes sign as well and, moreover, that there is a tendency to symmetry along the bisector at the corners of the cross-section. These similarities indicate that advection processes and turbulence processes are important with respect to the formation of the final shape of the outer bank cell, since large variation is seen in favouring and opposing (i.e. the sign of the source term agrees/disagrees with the sign of the vorticity) of the rotational sense of the cross-sectional flow in this area.

The features of the outer bank cell can be explained as follows. Due to anisotropy of the turbulence stresses, the profile of the streamwise velocities is deformed in the 
corners (cf. Demuren \& Rodi 1984; Nezu \& Nakagawa 1993). As a result, the $C F G$ term starts to play an important role in the outer bank region due to this deformation of the streamwise velocity field. Recall that the $C F G$-term just represents the vertical gradients in the streamwise velocity profile. The results in the steady state (figure 10) show that in the end the $C F G$-term favours the stable rotational sense that is observed in the flow field. The pattern of the $T U R B$-term, generally non-zero in corners of the flow, is opposed by advection of streamwise vorticity (the $A D V$-term). In this way, the $C F G$-term gets the opportunity to impose its rotational sense to the flow field and to make the streamlines get aligned with this rational sense as much as possible.

In the paper by Blanckaert \& de Vriend (2004), experimental findings are reported that point in the same direction. However, their experimental findings do not show the clear correlation of the $C F G$-term with the vorticity pattern on the one hand, and the distinctive influence of the $A D V$-term and the TURB-term on the other hand, as pronounced as the present LES results. This might be due to the fact that, in the present simulation, the cross-sectional geometry is very simple and that the flume is only mildly curved $(R / H=79)$, whereas in the experiment of Blanckaert \& de Vriend (2004) the bed was quite complex and the flume was more sharply curved $(R / H=18)$.

\subsection{Turbulence structure}

Within the context of the origin of the outer bank cell, only derivatives of the Reynolds shear stresses are of importance, for it is the derivatives that explicitly appear in the balance equation for $\omega_{\theta}$. The Reynolds shear stresses themselves are not important in this respect. However, these are interesting for understanding of the force field in each cross-section and the forces that are exerted on the solid walls. In this section, we focus on the principal stresses $\sigma_{1}$ and $\sigma_{2}$, which are in fact the true indicators for anisotropy, in view of Mohr's circle, be it in the two-dimensional cross-sectional plane. These principal stresses can be calculated from the original Reynolds stresses as follows:

$$
\sigma_{1,2}=\frac{\overline{u^{\prime 2}}+\overline{w^{\prime 2}}}{2} \pm \sqrt{\left(\frac{\overline{u^{\prime 2}}-\overline{w^{\prime 2}}}{2}\right)^{2}+\overline{u^{\prime} w^{\prime}}}
$$

Results for the difference between the principal stresses, $\sigma_{1}-\sigma_{2}$, and the original shear stresses $\overline{u^{\prime} w^{\prime}}$ are given in figure 11 for the $29^{\circ}$ cross-section and the $135^{\circ}$ cross-section.

Figure 11 clearly shows that the transverse and vertical velocity fluctuations are constrained by the geometry. At the free surface, vertical velocity fluctuations tend to zero, which causes the transverse velocity fluctuations to be dominant. At the outer bank, it is the other way around. As a result, the anisotropy is the most pronounced at the boundaries.

The pattern of the turbulent shear stresses $\overline{u^{\prime} w^{\prime}}$ at the $29^{\circ}$ cross-section (figure 11) shows a clear correlation with the observed circulation pattern. It can be seen in this figure that the outer bank circulation cell fully coincides with an area of negative $\overline{u^{\prime} w^{\prime}}$ (cf. Blanckaert \& Graf 2001). Apparently, the centre of the secondary flow cells coincides with a local extremum of the $\overline{u^{\prime} w^{\prime}}$ shear stresses. However, generally this does not need to be strictly the case, as at the $135^{\circ}$ cross-section a different behaviour is observed (figure $11 \mathrm{c}, d$ ). At the $135^{\circ}$ cross-section, two local extrema, both of comparable magnitude, are observed within the region where the outer bank cell is present.

According to Schwarz \& Bradshaw (1994), the efficiency of turbulent eddies in producing turbulent shear stresses can be roughly indicated by a structure parameter $a_{1}$, defined as the ratio of the magnitude of the turbulent shear stresses and twice the 

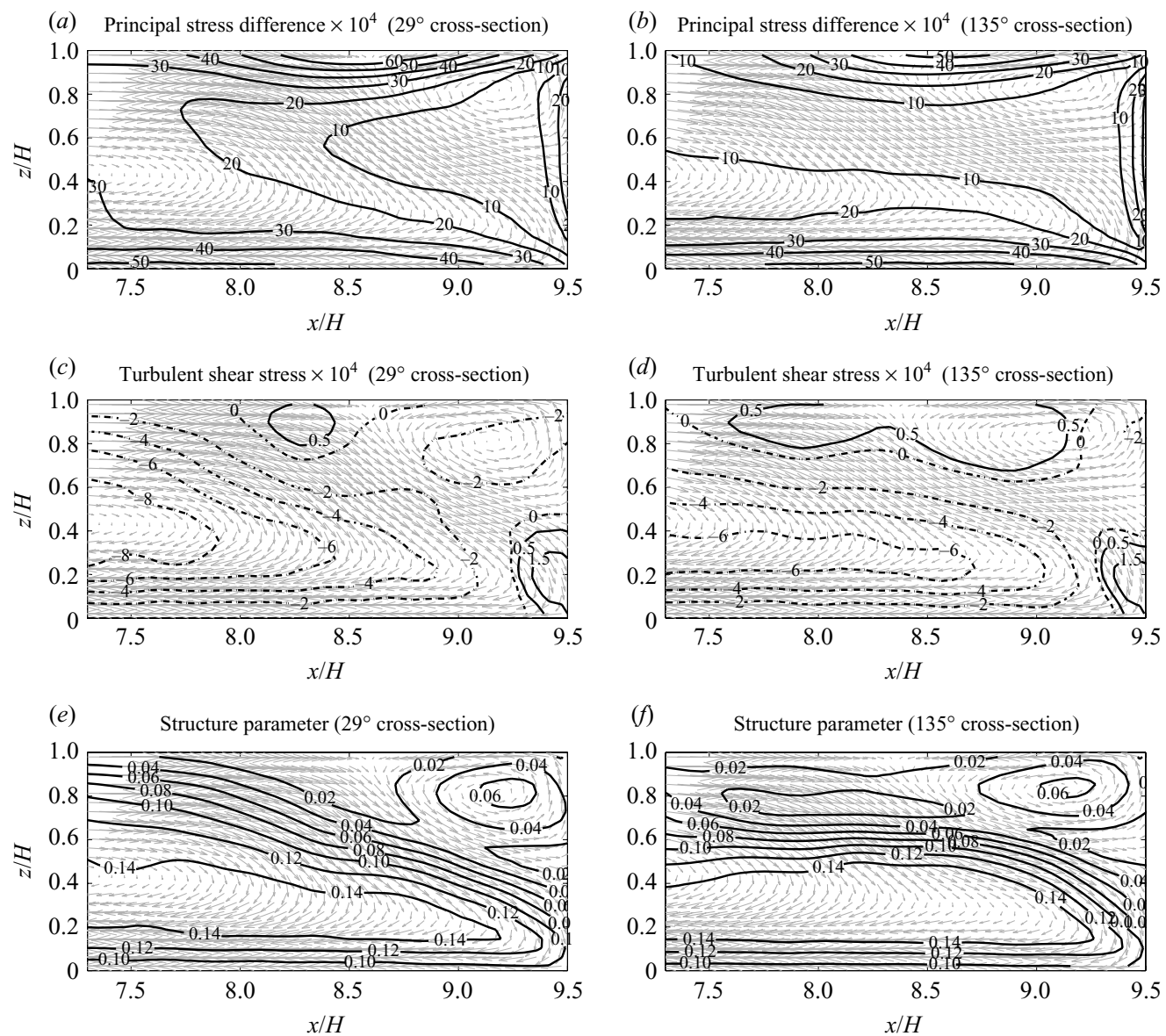

FiguRE 11. Principal stress difference $\left(\sigma_{1}-\sigma_{2}\right) \times 10^{4} / V_{a v}^{2}(a, b)$, shear stresses $\overline{u^{\prime} w^{\prime}} \times 10^{4} / V_{a v}^{2}$ $(c, d)$ and structure parameter $a_{1}(e, f)$ for the $29^{\circ}$ cross-section $(a, c$ and $e)$ and the $135^{\circ}$ cross-section $(b, d$ and $f)$.

amount of present turbulent kinetic energy,

$$
a_{1}=\frac{\sqrt{\overline{u^{\prime} w^{\prime}}+\overline{v^{\prime} w^{\prime}}}}{2 k},
$$

with $k=\frac{1}{2} \overline{u^{\prime} u^{\prime}}+\frac{1}{2} \overline{v^{\prime} v^{\prime}}+\frac{1}{2} \overline{w^{\prime} w^{\prime}}$. It can be shown for the case of straight open-channel flow, as done by Blanckaert \& de Vriend (2005), that this non-dimensional parameter $a_{1}$ is zero at the free surface, has a maximum of 0.14 almost halfway the water depth and a value of 0.10 at the bottom. The structure parameter $a_{1}$, resulting from the present LES results, is shown in figure 11(e, $f)$.

The values for $a_{1}$, given in figure 11, show that the deviations of the present curved open-channel flow from a straight open-channel flow are very small, since the theoretical values of 0.00 (at the free surface), 0.14 (halfway the water depth) and 0.10 at the bottom are also observed in the present results. The only difference is that the location of the maximum value 0.14 is found lower in the water column compared to the straight open-channel case. A remarkable observation from figure 11 is the clear footprint of the outer bank cell in the pattern of the structure parameter $a_{1}$. In this 
(a) Lumley triangle $\left(29^{\circ}\right.$ cross-section)

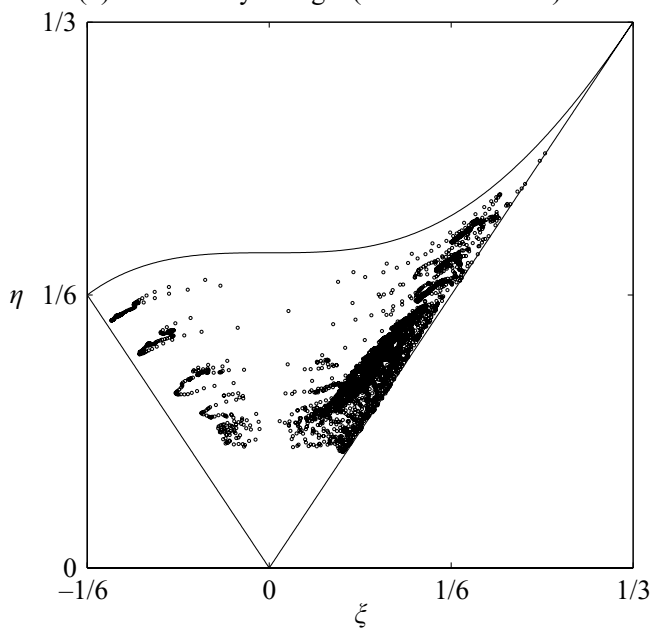

(b) Lumley triangle $\left(135^{\circ}\right.$ cross-section)

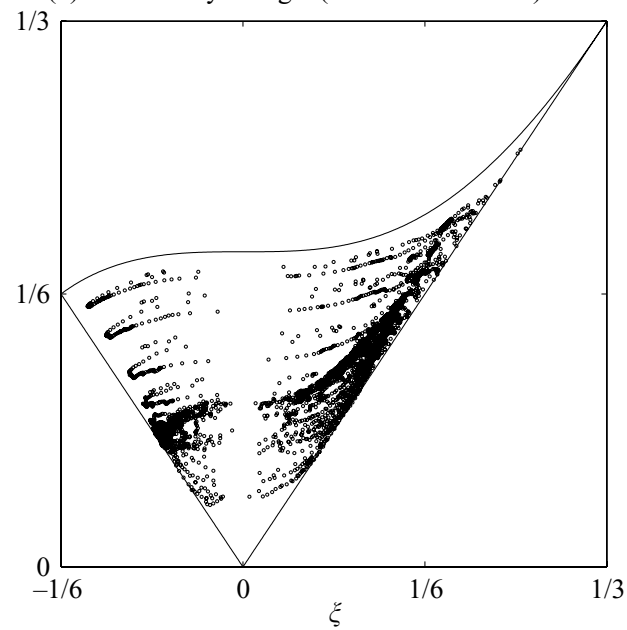

FIGURE 12. Lumley triangle for the $29^{\circ}$ cross-section (left panel) and for the $135^{\circ}$ cross-section (right panel).

outer bank region, a local increase of $a_{1}$ is found, reaching maximum values of about 0.06 .

The anisotropy of the Reynolds stresses can be studied by the invariants of the normalized anisotropy tensor $b_{i j}$ defined as

$$
b_{i j}=\frac{\overline{u_{i}^{\prime} u_{j}^{\prime}}}{\overline{u_{k}^{\prime} u_{k}^{\prime}}}-\frac{1}{3} \delta_{i j} .
$$

In fact, the structure parameter $a_{1}$ (3.5) is a variant of a specific element of this anistropy tensor $b_{i j}$ and can, therefore, be put in perspective by investigating the properties of $b_{i j}$. Because the trace of this tensor $b_{i j}$ is zero, it has only two independent invariants, denoted by $\xi$ and $\eta$. These two invariants are related to two eigenvalues, $\lambda_{1}$ and $\lambda_{2}$, by (see Pope 2000)

$$
\begin{aligned}
& \eta^{2}=\frac{1}{3}\left(\lambda_{1}^{2}+\lambda_{1} \lambda_{2}+\lambda_{2}^{2}\right), \\
& \xi^{3}=-\frac{1}{2} \lambda_{1} \lambda_{2}\left(\lambda_{1}+\lambda_{2}\right) .
\end{aligned}
$$

These invariants, $\xi$ and $\eta$, can be graphically shown in an anisotropy invariant map (see Lumley 1978), often referred to as the Lumley triangle. A detailed interpretation of the Lumley triangle is given by Simonsen \& Krogstad (2005).

The values for $\xi$ and $\eta$ for the $29^{\circ}$ and $135^{\circ}$ cross-sections are shown in figure 12 within the context of the Lumley triangle. For the $29^{\circ}$ cross-section, it is seen that the greater part of the values is found near the right straight side of the triangle, where the state of the turbulence can be characterized as axisymmetric with one dominant eigenvalue. Axisymmetric turbulence means that two of the principal stresses $\sigma_{i}$ are equal. Generally, the $\xi, \eta$ values found in the log-law region of a turbulent boundarylayer flow are at this side of the triangle (see Liu \& Pletcher 2008). For axisymmetric turbulence with one large eigenvalue, the shape of the stress tensor is a prolate spheroid. For the $29^{\circ}$ cross-section, the values near the right straight side of the triangle are found in the lower half of the water column, up to $z / H \approx 0.7$, and near the sidewalls of the cross-section. 
(a) Main kinetic energy $\left(29^{\circ}\right.$ cross-section)

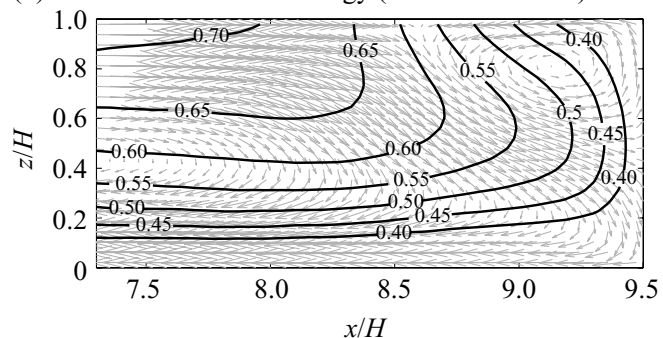

(c) Turbulent kinetic energy $\times 10^{3}\left(29^{\circ}\right.$ cross-section $)$

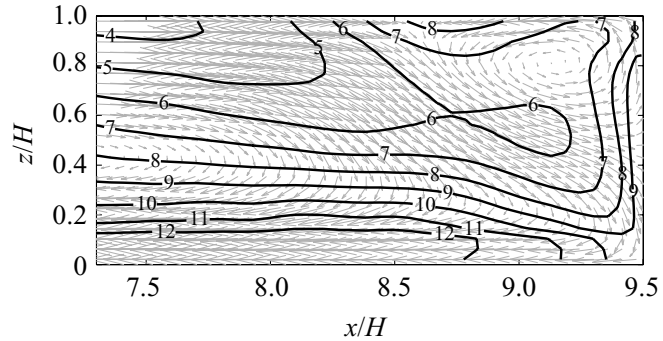

(b) Main kinetic energy $\left(135^{\circ}\right.$ cross-section)

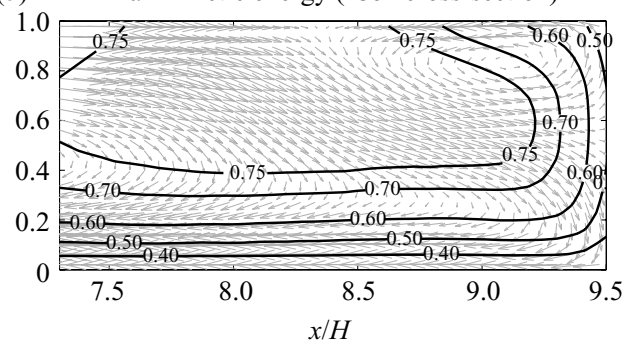

(d) Turbulent kinetic energy $\times 10^{3}\left(135^{\circ}\right.$ cross-section $)$

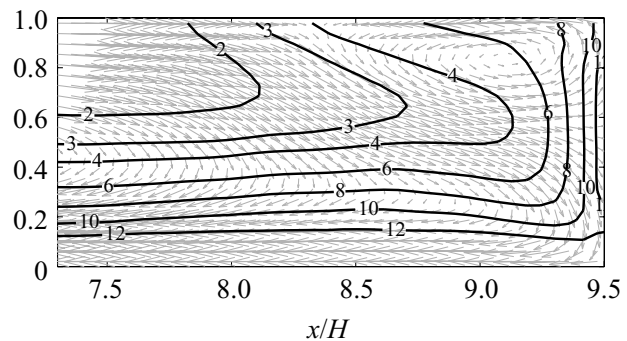

FIGURE 13. Total kinetic energy $(a, b)$ and turbulent kinetic energy $\left(\times 10^{3}\right)(c, d)$ for the $29^{\circ}$ cross-section $(a, c)$ and the $135^{\circ}$ cross-section $(b, d)$. The values are non-dimensionalized by $V_{a v}^{2}$.

In the middle of the $29^{\circ}$ cross-section near the free surface, the $\xi, \eta$ values are found near the left straight side of the triangle. At this side, the state of the turbulence can be characterized as axisymmetric with one small eigenvalue. For this type of turbulence, the shape of the stress tensor is an oblate spheroid. Moving from the inner bank to the outer bank, the $\xi, \eta$ values move from the right side of the triangle to the left, and move towards the curved top side of the triangle, when approaching the free surface. This curved side of the triangle is associated with two-component turbulence.

The $\xi, \eta$ values for the $135^{\circ}$ cross-section show more or less the same behaviour as for the $29^{\circ}$ cross-section. However, the $\xi, \eta$ values for the $135^{\circ}$ cross-section are more uniformly distributed over the triangle. It is remarkable that the $\xi, \eta$ values tend to approach the origin of the triangle, which is associated with isotropic turbulence. These values are found in the outer half of the cross-section around $z / H \approx 0.75$.

Considering the Lumley triangle for the $29^{\circ}$ and $135^{\circ}$ cross-sections, it can be concluded that in the core region of the flow (i.e. far from the walls), the turbulence is axisymmetric with one small eigenvalue of the anisotropy tensor $b_{i j}$. This turbulence state is favoured far downstream of the inlet of the bend, with a weak tendency to isotropy in the outer half of the cross-section.

\subsection{Kinetic energy}

Figure 13 shows the distribution of the main kinetic energy, defined as $\frac{1}{2} U_{i} U_{i}$, and the turbulent kinetic energy (TKE), defined as $\frac{1}{2} \overline{u_{i}^{\prime} u_{i}^{\prime}}$, for the $29^{\circ}$ and $135^{\circ}$ cross-sections. The plots of the main kinetic energy reflect the outward shift of high momentum fluid due to differential advection, since it can be seen that the location of the peak value of the total kinetic energy has shifted towards the outer bank at the $135^{\circ}$ cross-section compared to the $29^{\circ}$ cross-section.

The graph of the turbulent kinetic energy shows a strong increase towards the solid walls for both the $29^{\circ}$ and $135^{\circ}$ cross-sections, which is also apparent in straight 
open-channel flows (cf. Nezu \& Nakagawa 1993). Remarkably enough, Blanckaert \& Graf (2001) found a different picture of the TKE. Besides the increase of TKE towards the solid walls, they also found an even stronger increase of TKE towards the free surface in the core region of the flow, which is not explicitly explained in their paper and absent in the present LES results. The profile of the TKE in the core region of the flow, as it results from the present LES, much resembles its straight flow equivalent (cf. Nezu \& Nakagawa 1993). Furthermore, it is remarkable that there is a local increase of TKE visible in the region covered by the outer bank circulation cell. Apparently, the outer bank cell region is a region of increased turbulence activity.

\subsection{Production of turbulent kinetic energy}

The simulation of real river systems, especially when sediment transport and morphodynamics are considered, requires the use of RANS simulations instead of large-eddy simulations in view of the computational costs. Many RANS solvers used in practice make use of an isotropic eddy viscosity model such as the standard $k-\epsilon$ turbulence closure model. The basis of these isotropic eddy viscosity models is the assumption of a linear dependency of the Reynolds stresses on the rate-of-strain tensor, with a certain scalar eddy viscosity as a factor of proportionality.

However, Booij (2003) has shown that these models are unable to reproduce the $\overline{u^{\prime} v^{\prime}}$ stresses of the correct sign in single-bend flows. Booij (2003) has mentioned that this would require a negative eddy viscosity and has also simulated an open-channel bend with an RANS model with a linear $k-\epsilon$ turbulence closure model.

Moreover, these models have been proved not to be able to reproduce secondary flow of Prandtl's second kind (cf. Demuren \& Rodi 1984 and Speziale 1987), as it is the anisotropy of the turbulence stresses that makes the secondary flow to come into existence. Blanckaert \& de Vriend (2004) contribute to the discussion that it is possible to reproduce non-zero turbulence anisostropy (i.e. $\overline{u^{\prime 2}}-\overline{w^{\prime 2}} \neq 0$ ) with an isotropic eddy viscosity model, and that it is not possible to represent fluxes of turbulent kinetic energy from the turbulent motion to the main motion. Blanckaert \& de Vriend (2004) argue that some perturbations of the flow, representing a secondary flow cell of Prandtl's second kind, would dampen out because isotropic eddy viscosity models are absolutely dissipative.

The present LES results enable the analysis of the occurring fluxes of turbulent kinetic energy, by the calculation of the production term $P$, arising with opposite signs in the balance equation for mean and turbulent kinetic energy. This term $P$ reads

$$
P=-\frac{1}{2}\left(\overline{u_{i}^{\prime} u_{j}^{\prime}}-\frac{2}{3} k \delta_{i j}\right) \overline{S_{i j}},
$$

with $k=\frac{1}{2} \overline{u_{i}^{\prime} u_{i}^{\prime}}, i, j=r, \theta, z, \delta_{i j}$ the Kronecker delta and $\overline{S_{i j}}$ the rate-of-strain tensor, based on the mean velocities containing the components

$$
\begin{gathered}
\overline{S_{r r}}=2 \frac{\partial U}{\partial r}, \overline{S_{\theta \theta}}=2\left(\frac{1}{r} \frac{\partial V}{\partial \theta}+\frac{U}{r}\right), \overline{S_{z z}}=2 \frac{\partial W}{\partial z}, \\
\overline{S_{\theta r}}=\overline{S_{r \theta}}=\frac{\partial V}{\partial r}+\frac{1}{r} \frac{\partial U}{\partial \theta}-\frac{V}{r}, \overline{S_{\theta z}}=\overline{S_{z \theta}}=\frac{1}{r} \frac{\partial W}{\partial \theta}+\frac{\partial V}{\partial z}, \overline{S_{z r}}=\overline{S_{r z}}=\frac{\partial U}{\partial z}+\frac{\partial W}{\partial r} .
\end{gathered}
$$

In this context, the overbar indicates that time-averaged quantities are concerned. Two types of fluxes are considered now: the total TKE flux (with $i, j=r, \theta, z$ ) and the cross-sectional TKE flux (with $i, j=r, z$ ). The total TKE flux and the cross-sectional TKE flux are shown in figure 14 for the $29^{\circ}$ cross-section and the $135^{\circ}$ cross-section. 
(a)

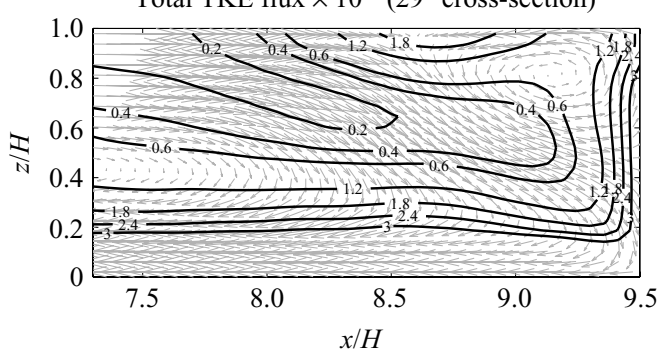

(c) Crosssectional TKE flux $\times 10^{3}\left(29^{\circ}\right.$ cross-section $)$

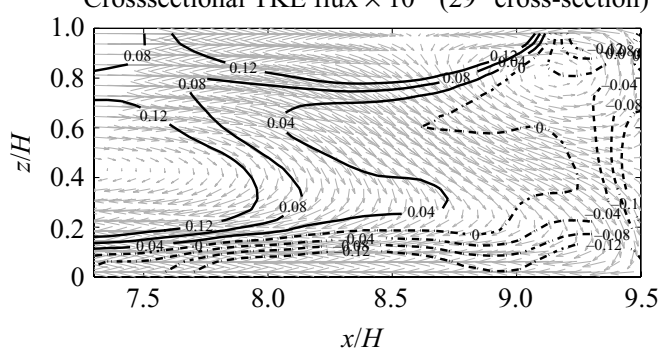

(b)

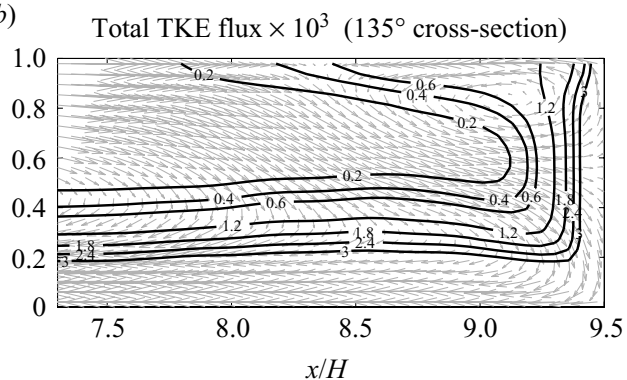

(d) Crosssectional TKE flux $\times 10^{3}\left(135^{\circ}\right.$ cross-section)

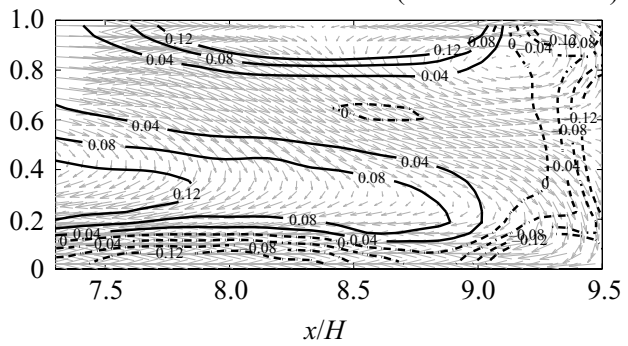

Figure 14. Total TKE flux $\left(\times 10^{3} H / V_{a v}^{3}\right)(a, b)$ and cross-sectional TKE flux $\left(\times 10^{3} H / V_{a v}^{3}\right)$ $(c, d)$ at the $29^{\circ}$ cross-section $(a, c)$ and the $135^{\circ}$ cross-section $(b, d)$.

In figure 14, it can be seen that the total production of TKE is calculated to be positive everywhere in the outer bank region. Hence, there is only one-way transfer of TKE from mean motion to the turbulent motion. Moreover, it is observed that the total production of TKE increases towards the solid wall. It is remarkable that in the area where the outer bank cell is present, the total production of TKE also increases, be it up to about three times higher values at the $29^{\circ}$ cross-section compared to the $135^{\circ}$ cross-section.

In figure 14, it is also seen that the cross-sectional fluxes of TKE are generally one order of magnitude smaller than the total TKE fluxes. Within the region covered by the primary circulation cell, the maximum values are found in the lower part of the water column, whereas minimum values are found at the free surface and the bottom. Negative values of the cross-sectional fluxes of TKE are found at the bottom and sidewall in the outer bank region (see figure 14) and at the lower part of the inner bank (not shown here). These negative values are associated with a flux of TKE from the turbulent motion to the main motion. Figure 14 also shows a sort of transition area between the primary circulation cell and the counter-rotating outer bank cell $(x \approx 8.4 H, z \approx H)$, where relatively high values are found for the cross-sectional TKE flux. The values, found in this area, are of the same order of magnitude as the values in the core of the cross-section, and indicate that relatively much TKE is transported from the main flow towards the turbulent motion.

It is the occurrence of negative values of the cross-sectional flux of TKE that explains why the outer bank cell cannot be reproduced by RANS models with an isotropic eddy viscosity turbulence closure model (cf. Booij 2003). As mentioned before, the distribution of the turbulence stresses on the one hand and centrifugal effects on the other cause the existence of the outer bank cell. Although isotropic eddy viscosity models, in principle, are able to reproduce anisotropy of turbulent stresses, i.e. $\overline{u^{\prime 2}}-\overline{w^{\prime 2}} \neq 0$, these models are not able to reproduce fluxes of TKE from the 

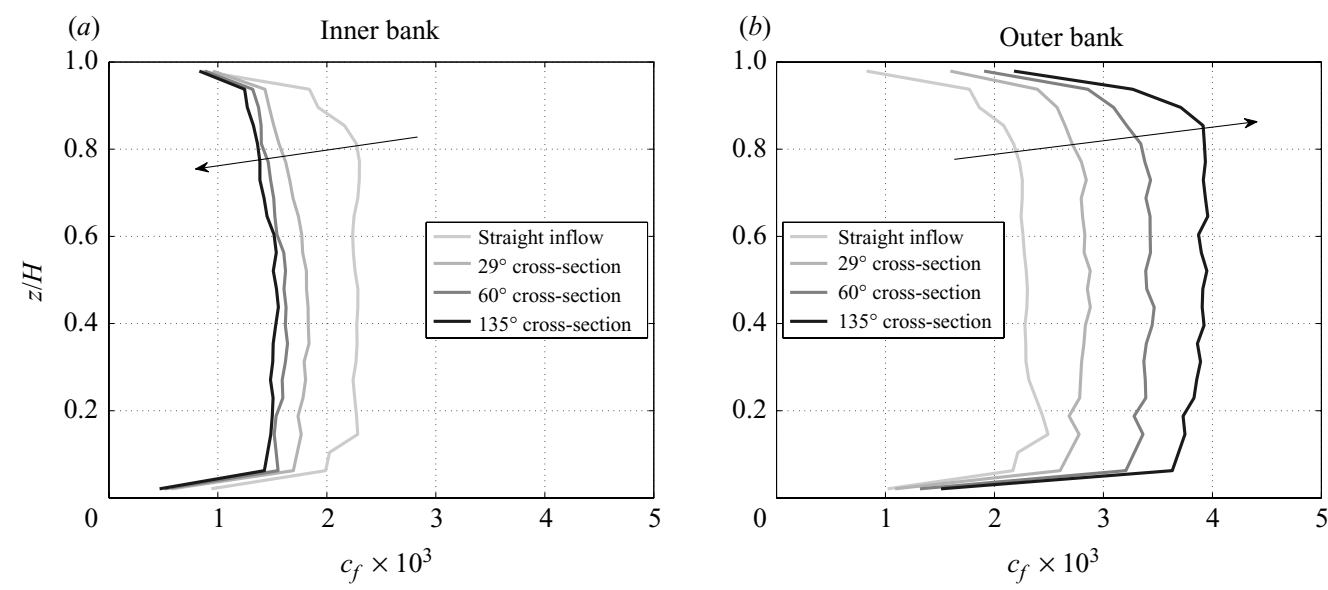

FIGURE 15. Wall shear stresses on the inner bank $(a)$ and the outer bank $(b)$, given by $c_{f}$ and multiplied by $10^{3}$. The arrow denotes the tendency when moving downstream.

turbulent motion to the main motion, which are obviously present in the outer bank cell region. With just dissipation of TKE, the counter-rotating outer bank cell would therefore damp out. These findings are consistent with the experimental findings of Blanckaert \& de Vriend (2004), as well as with the results of the simulations of Kimura et al. (2008), who simulated curved open-channel flow using a nonlinear $k-\epsilon$ model.

\subsection{Wall shear stresses}

Wall shear stresses are of particular interest, for instance in the context of engineering or geomorphology, since these are the measure for the forces that are exerted on the wall. In river engineering, the wall shear stresses can be directly related to sedimentation and erosion processes.

The total wall shear stresses, $\tau_{w}$, are non-dimensionalized by the bulk velocity squared, $V_{a v}^{2}$, thus defining the skin-friction coefficient $c_{f}$. The distributions of the bank shear stresses, expressed in terms of $c_{f}$, are shown in figure 15 for four different cross-sections along the flume. The distribution of the bed shear stresses is shown in figure 16 for the same four cross-sections. From the pressure difference between the inlet and the outlet of the domain, a flume-averaged $c_{f}$ value can be calculated. This value was found to be $c_{f}=3.8 \times 10^{-3}$ and can be used as a reference value.

In figure 15, it is seen that at the straight inflow section (1 m upstream of the start of the bend) the distribution and magnitude of the bank shear stresses are quite the same for the inner bank and outer bank. Downstream in the bend, the bank shear stresses gradually decrease/increase along the inner/outer bank, thus reflecting the influences of differential advection. In the far field, the outer bank shear stresses reach values of about 2.5 times higher than the inner bank shear stresses.

The distribution of the bed shear stresses (figure 16) at the straight inflow section is comparable with distributions that are commonly observed for straight open-channel flows (see, for instance, Yang 2005). However, moving downstream the bed shear stresses strongly increase in the outer bank region up to $50 \%$ higher values compared to the straight inflow cross-section. In the inner bank region, the bed shear stresses increase in the near field of the bend, but decrease in the far field of the bend. 


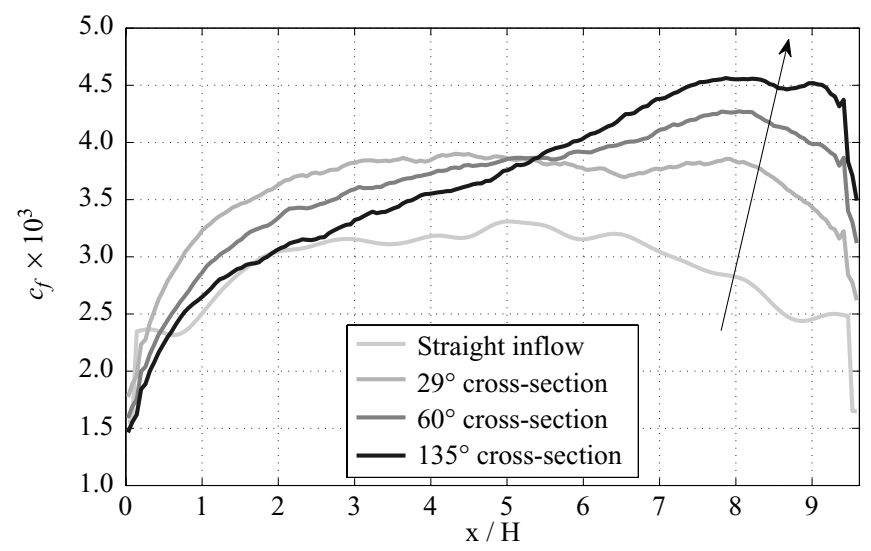

FIGURE 16. Bed shear stresses, expressed by $c_{f}$ multiplied by $10^{3}$. The arrow denotes the tendency when moving downstream.

Figure 16 clearly shows how the distribution of the bed shear stresses is deformed due to curvature influences. In the far field of the bend, the distribution of the bed shear stresses is approximately linearly varying across the width. But it is also seen that over the whole bend, the bed shear stresses significantly increase compared to the straight inflow section upstream. This entire increase of the bed shear stresses is a result of secondary currents and the redistribution of momentum due to curvature.

The observation that curvature makes the wall shear stresses increase is very relevant from a flood protection point of view, because increased resistance to the flow due to higher energy losses leads to a reduced conveyance capacity in real river systems.

\subsection{Sensitivity to changes in the subgrid-scale model and the wall model}

In this section, the results are shown for Runs 2-5. Recall that in these simulations axisymmetric flow is considered, which can be physically associated with the flow in the far field of the experimental flume (see figure 5). The mutual differences between Runs 2-5 are the choices for the subgrid-scale model and the wall model (see table 2).

Compared to the spatially developing flow case (Run 1), the axisymmetric flow case (Runs 2-5) is much more suitable for an investigation of the influence of changes in the subgrid-scale model and the wall model. This is because of its low computational costs due to the possibility to use periodic boundary conditions in streamwise direction.

A snapshot of the instantaneous transverse turbulent velocities is given in figure 17 for Run 2. In this figure, the bicellular pattern of the secondary flow can be readily seen at the free surface. In figure 17, it can also be seen that the transverse velocities at the free surface at the two secondary flow cells are of the same order of magnitude.

From figure 18, to be compared with figure $9(d)$, it can be seen that the incorporation of the local pressure gradient hardly influences the results of the simulation. The differences that can be observed in figure 18 can mainly be attributed to the choice of the subgrid-scale model. The dynamic Smagorinsky model tends to favour the centre region cell of secondary flow, as Runs 4 and 5 show higher values of the streamfunction, compared to Runs 2 and 3. 


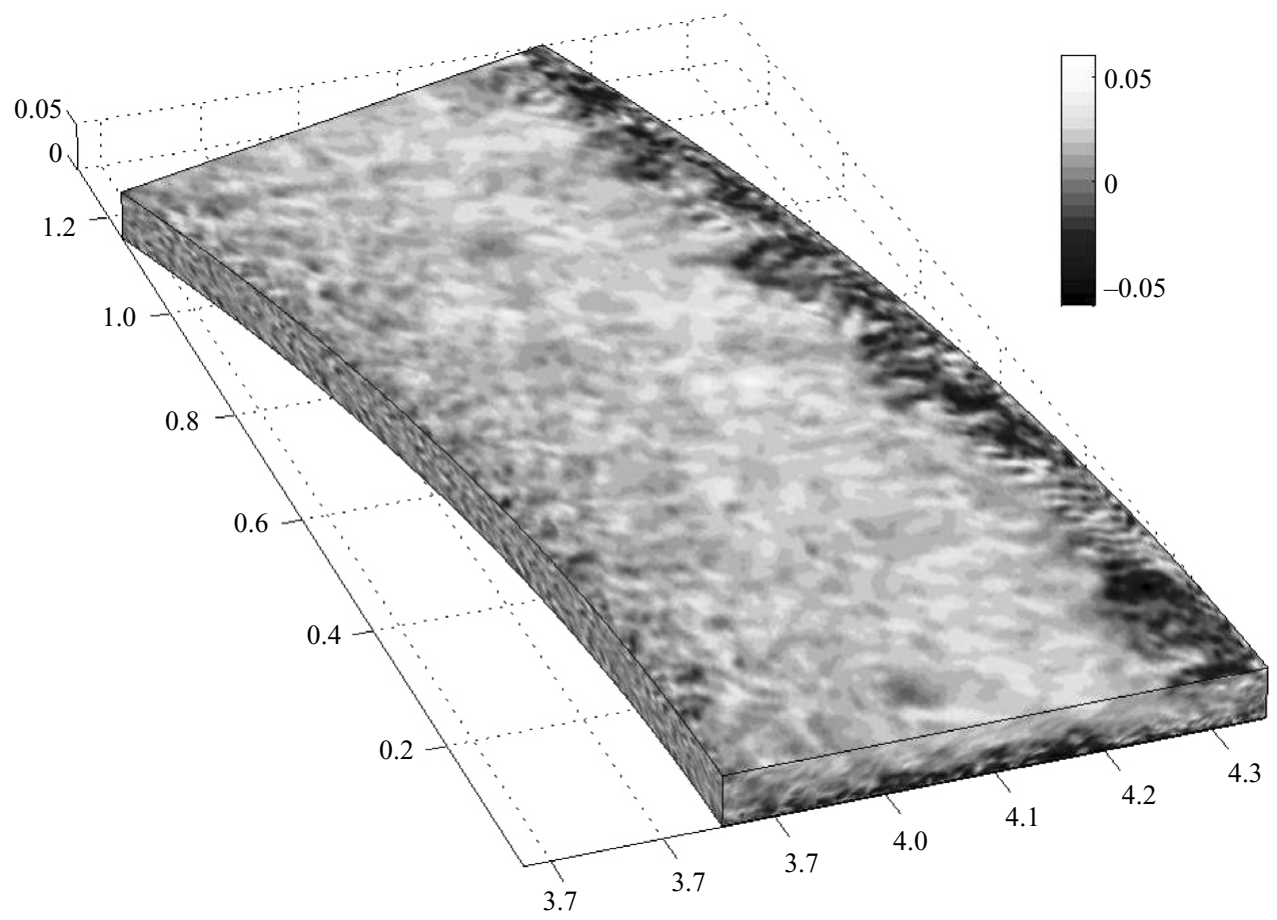

FIGURE 17. Snapshot of the transverse velocities for Run 2. Distances are in metres and velocities are in metres per second.
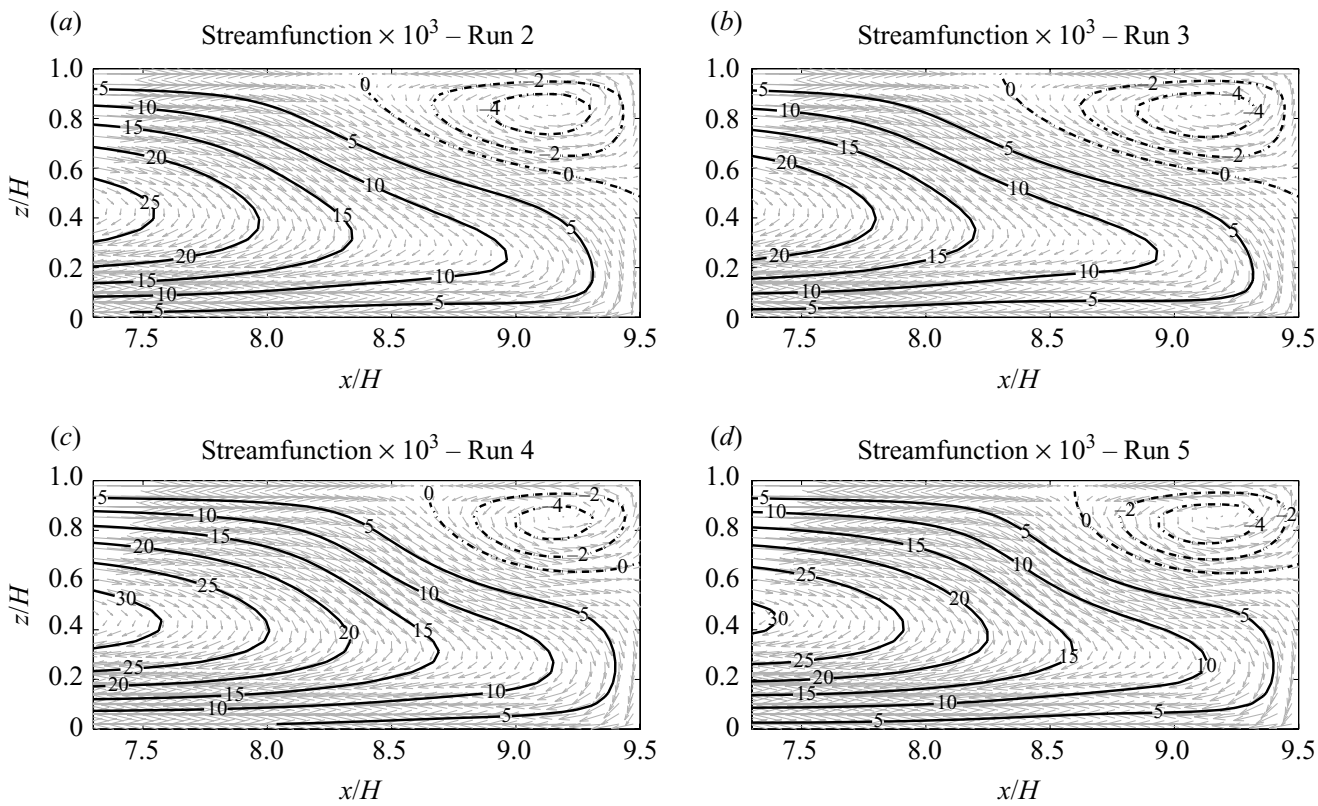

FIGURE 18. Streamfunction $\psi \times 10^{3} / H R V_{a v}$ for Runs 2, 3, 4 and 5, in lexicographic ordering. Notice that only the outer $23 \%$ of the cross-section is shown. 

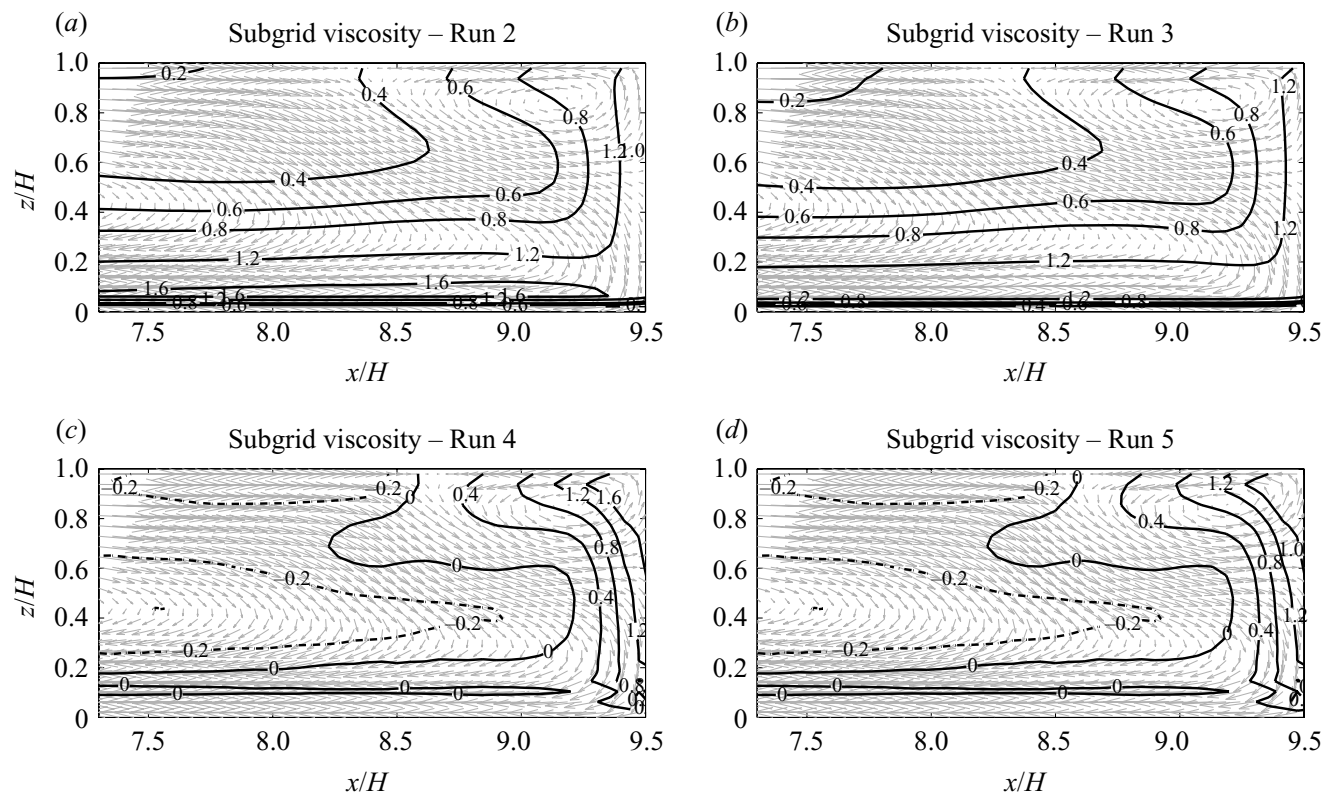

FIGURE 19. Distribution of the subgrid-scale viscosity (in $\mathrm{m}^{2} \mathrm{~s}^{-1}$ and multiplied by $10^{6}$ ) for Runs 2, 3, 4 and 5 in lexicographic ordering.

Nonetheless, the mutual differences between Runs 2, 3, 4 and 5 are small enough to justify the use of only the simple turbulence modelling tools for the subgrid-scale stresses and the near-wall region for the present single-bend flow.

It is observed from figure 18 that, as soon as the primary secondary flow cell (solid lines) is overestimated, the outer bank cell (dash-dotted lines) is underestimated. This is particularly visible for Run 4 and Run 5, where the outer bank cell gets smaller, whereas the primary circulation cell gets stronger. The interrelation between the primary secondary flow cell and the counter-rotating outer bank cell can be explained by two mutually opposing differential advection mechanisms: on the one hand, we have the large primary flow cell that advects high momentum fluid outwards and, on the other hand, we have the small outer bank cell that advects low momentum fluid inwards.

In figure 19, the distribution of the subgrid-scale viscosity $v_{s g s}$ is shown for the outer bank region for each run. The molecular viscosity $v_{m o l}$ is equal to $1.0 \times 10^{-6}$ $\mathrm{m}^{2} \mathrm{~s}^{-1}$. For Run 2 and Run 3, the subgrid-scale viscosity $v_{s g s}$ is of the same order of magnitude as the molecular viscosity $v_{m o l}$. The values of $v_{s g s}$ are high near the wall, but vanish at the wall due to the Van Driest damping.

In the outer bank region of the cross-section, the values of the subgrid-scale viscosity are of the same order of magnitude for each run. However, in the region of the primary circulation cell, Run 4 and Run 5 show a negative subgrid-scale viscosity $v_{s g s}$. This negative eddy viscosity is associated with backscatter of turbulent kinetic energy from the sub-grid scale to the supra-grid scale, not to be confused with backscatter of turbulent kinetic energy from the turbulent motion to the mean motion.

The observation that the standard and dynamic Smagorinsky models do not give much different results is of large importance for the possible use of large-eddy simulation for real river systems in the future. For those cases, the use of the standard 
Smagorinsky model can be beneficial since it is computationally cheaper than the dynamic Smagorinsky model.

\section{Summary and conclusions}

This paper reports a detailed numerical information on the physics of the flow through a curved laboratory flume which has been obtained by means of large-eddy simulation. The focus of the paper is mainly on the features and origin of the secondary flow patterns and the turbulence structure of the flow. A good agreement with published experimental results is achieved. Although large-eddy simulation has become rather common for the simulation of straight open-channel flow, this is (to the knowledge of the authors) the first large-eddy simulation of a single-bend open-channel flow that is reported in the literature.

The main contribution of the present results to the understanding of the physics of curved open-channel flow is fourfold. Firstly, the present paper gives an explanation of the origin the secondary flow currents in a curved open-channel flow. Comparable investigations have been carried out for straight open-channel flow in the past, but not for curved open-channel flow as done with the level of accuracy of the presented results. In straight open-channel flow, secondary currents near the banks are due to turbulence anisotropy (circulation of Prandtl's second kind), whereas in curved openchannel flow the secondary flow near the outer bank is a result of both turbulence anisotropy and centrifugal effects (strictly neither of Prandtl's first or second kind).

Secondly, aspects of turbulence anisotropy are studied by means of the principal stresses in the cross-sectional plane and by means of the turbulence anisotropy tensor in the three dimensions. In the far field a tendency to isotropic turbulence is observed in the outer half of the cross-section. With respect to the energy of the turbulent motion, it was found that its content as well as its production is locally increased in the region covered by the outer bank cell.

Thirdly, the paper reflects on the use of RANS models with an isotropic eddy viscosity for the simulation of curved open-channel flows. These models are quite commonly used in the river engineering practice. The results stress the need of a negative eddy viscosity in order to properly reproduce turbulence stresses of the correct sign and underline that the observed transfer of turbulent kinetic energy is incompatible with the isotropic character of linear turbulence closure relations.

Fourthly, the results for the wall shear stresses show how the distribution of the wall shear stresses is deformed compared to straight open-channel flow. The bed shear stresses deform from a symmetric profile to a linearly varying profile in the far field, yielding up to $50 \%$ higher values in the outer bank region. A similar increase is observed for the bank shear stresses at the outer bank. Moreover, the results show that due to curvature the distribution of the bed shear stresses as a whole is amplified compared with straight open-channel flow. These results, presented for the wall shear stresses, also contribute to the current state of knowledge since these are difficult to measure experimentally.

Since only simple turbulence modelling techniques are used (e.g. the standard Smagorinsky model), the sensitivity of the results to changes in the subgrid-scale model and the wall model is investigated by means of a separate simulation of the axisymmetric part of the flow in the far field of the bend using periodic boundaries. It turned out that differences are present, but they are small enough not to change the conclusions of this paper. 


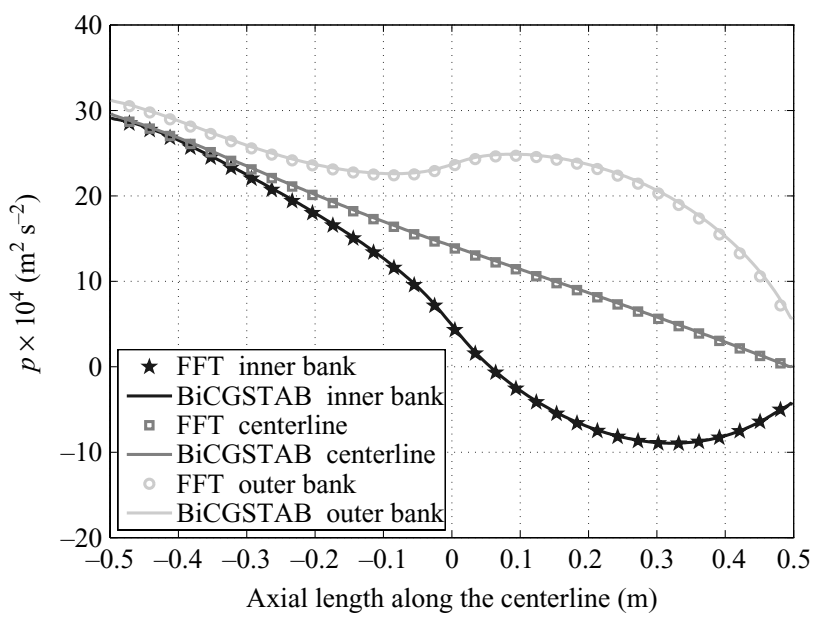

Figure 20. Depth-averaged pressure (multiplied by $10^{4}$ ) along the inner bank, centreline and outer bank of the geometry. The signs represent the data from the FFT-based simulation, while the solid lines represent the data from the BiCGSTAB-based simulation. Negative values on the horizontal axis are in the straight section and positives values are in the curved section.

This work is supported by the Dutch Technology Foundation, applied science division of NWO and the Technology Programme of the Ministry of Economic Affairs. Robert Jan Labeur is acknowledged for some help with the implementation of the ILU-BiCGSTAB method.

\section{Appendix A. Poisson solver}

In $\S 2.4$, we claim that the term $1 / r \cdot \partial p / \partial r$ in (2.14) is negligible for our particular computation. In order to support this claim, two additional simulations are defined. The first simulation makes use of the FFT-based method for the pressure Poisson equation under the assumption that the term $1 / r \cdot \partial p / \partial r$ is negligible. The second simulation makes use of the BiCGSTAB-based method for the pressure Poisson equation without any assumption.

The new computational domain is again based on the geometry shown in figure 5 . However, the length of the straight inflow section is taken $0.5 \mathrm{~m}$ and the length of the curved section is also taken $0.5 \mathrm{~m}$ (along the channel centreline). In this case, there is no straight outflow section. This small computational domain is chosen in order to keep the computational costs low. The mesh contains $168 \times 336 \times 24$ grid cells in transverse, streamwise and vertical direction, respectively $(1.4$ million grid points in total). The hydraulic parameters are shown in table 1.

The methodology is the same as for the previous simulations described in the paper: after the velocity field has reached its statistically steady state, the velocity field is averaged over a certain time to obtain smooth flow statistics. At this point, we focus only on the output for the pressure $p$. Recall that the constant density $\rho$ is absorbed in the pressure.

For the comparison of the results, we apply depth averaging on the time-averaged results for the pressure $p$. The values for the depth-averaged pressure along the inner bank, centreline and outer bank of the geometry are shown in figure 20. In this figure, it can be seen that the results from both the FFT-based simulation and the BiCGSTAB-based simulation are approximately identical. The mass-loss, associated 

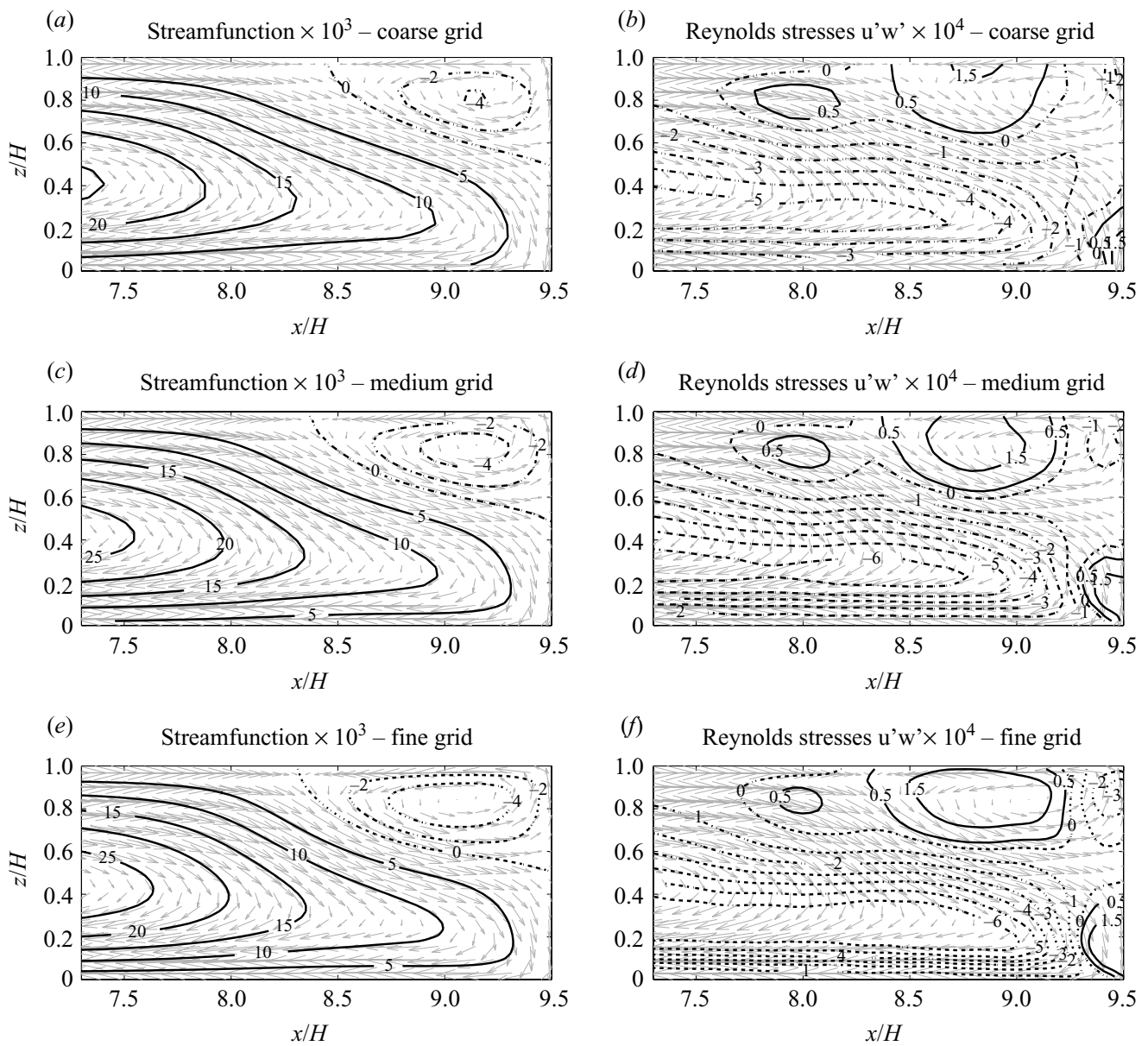

FIGURE 21. $(a, c, e)$ : The streamfunction $\psi \times 10^{3} / H R V_{a v}$ for the coarse, medium and fine mesh $(a-f) .(b, d, f)$ : The Reynolds stresses $\overline{u^{\prime} w^{\prime}} \times 10^{4} / V_{a v}^{2}$ for the coarse, medium and fine mesh $(a-f)$.

with leaving out the term $1 / r \cdot \partial p / \partial r$, can be calculated in terms of the discharge as $Q_{\text {in }}-Q_{\text {out }} \approx 10^{-8} \cdot Q_{\text {in }}$, which is certainly negligible. For this computational set-up, the whole flow solver was accelerated with a factor of about 7.

\section{Appendix B. Mesh independency}

In order to prove that the solutions of the presented simulations are independent of the mesh, Run 2 from the paper is chosen to be simulated on two other meshes: a coarser mesh and a finer mesh. Recall that Run 2 is the simulation of the axisymmetric flow in the far field of the flow set-up (see figure 5). The big advantage of axisymmetric flow is that periodic boundary conditions can be applied in streamwise direction, thus saving much computational time. An instantaneous result of Run 2, shown in figure 17, also shows the dimensions of the computational domain.

The simulations are run on different meshes: a coarse mesh $(112 \times 200 \times 16)$, a medium mesh $(168 \times 300 \times 24)$ and a fine mesh $(252 \times 460 \times 36)$. The results for the streamfunction $\psi$ and the Reynolds stresses $\overline{u^{\prime} w^{\prime}}$ of the three simulations are shown in figure 21 for the outer bank region. 
In the background of the pictures in figure 21 the velocity vectors are shown. For this purpose, the velocity fields of the medium and the fine mesh are interpolated to the grid of the coarse mesh to make the comparison comprehensible. It is clearly seen in figure 21 that the differences between the results of the three simulations are quite minimal.

\section{REFERENCES}

Balaras, E., Benocci, C. \& Piomelli, U. 1996 Two-layer approximate boundary conditions for large-eddy simulations. AIAA J. 34, 1111-1119.

Bathurst, J. C., Thorne, C. R. \& Hey, R. D. 1977 Direct measurements of secondary currents in river bends. Nature 269, 504-506.

BlancKaert, K. \& Graf, W. H. 2001 Mean flow and turbulence in open-channel bend. J. Hydr. Engng 127, II.1-II.13.

BlancKaert, K. \& De VRiend, H. J. 2004 Secondary flow in sharp open-channel bends. J. Fluid Mech. 498, 353-380.

BlancKaeRT, K. \& DE VRIEND, H. J. 2005 Turbulence characteristics in sharp open-channel bends. Phys. Fluids 17, 055102.

Blokland, T. 1985 Turbulence measurements in a curved flume (in Dutch). MSc thesis, Delft University of Technology.

Bools, R. 2003 Measurements and large-eddy simulations of the flows in some curved flumes. J. Turbulence 4, 1-17.

Bradshaw, P. 1987 Turbulent secondary flows. Ann. Rev. Fluid Mech. 19, 53-74.

Broglia, R., Pascarelli, A. \& Piomelli, U. 2003 Large-eddy simulations of ducts with a free surface. J. Fluid Mech. 484, 223-253.

BRUNDETT, E. \& BAINES, W. D. 1964 The production and diffusion of vorticity in duct flow. J. Fluid Mech. 19, 375-394.

Calhoun, R. J. \& Street, R. L. 2001 Turbulent flow over a wavy surface: neutral case. J. Geoph. Res. 106, 9277-9293.

Demuren, A. O. \& RoDI, W. 1984 Calculation of turbulence-driven secondary motion in non-circular ducts. J. Fluid Mech. 140, 189-222.

Einstein, H. A. \& Harder, J. A. 1954 Velocity distribution and the boundary layer at channel bends. Trans. $A G U$ 35, 114-120.

Germano, M., Piomelli, U., Moin, P. \& CABot, W. H. 1991 A dynamic subgrid-scale eddy viscosity model. Phys. Fluids A 3, 1760.

Gessner, F. B. 1973 The origin of secondary flow in turbulent flow along a corner. J. Fluid Mech. 59, $1-25$.

Gessner, F. B. \& Jones, J. B. 1965 On some aspects of fully developed turbulent flow in a rectangular channel. J. Fluid Mech. 23, 689-713.

GustafsSON, I. 1978 A class of first order factorization methods. BIT 18, 142-156.

Hicks, F. E., Jin, Y. C. \& StefFler, P. M. 1990 Flow near sloped bank in curved channel. J. Hydr. Engng 116, 55-70.

Kimura, I., UijtTewaal, W. S. J., van Balen, W. \& Hosoda, T. 2008 Application of the nonlinear $k-\epsilon$ model for simulating curved open channel flows. In Proc. Riverflow 2008, pp. 99-108. Kubaba.

Koken, M. \& Constantinescu, G. 2008 An investigation of the flow and scour mechanisms around isolated spur dikes in a shallow open channel: 2. Conditions corresponding to the final stages of the erosion and deposition process. Water Resour. Res. 44, W08407.

Lilly, D. K. 1992 A proposed modification of the germano subgrid-scale closure method. Phys. Fluids A 4, 633.

Liu, K. \& Pletcher, R. H. 2008 Anisotropy of a turbulent boundary layer. J. Turbulence 9, 1-18.

Lumley, J. 1978 Computational modelling of turbulent flows. Adv. Appl. Mech. 18, 123.

McCoy, A., Constantinescu, G. \& Weber, L. J. 2008 Numerical investigation of flow hydrodynamics in a channel with a series of groynes. J. Hydr. Engng 134, 157-172.

Nezu, I. \& Nakagawa, H. 1993 Turbulence in Open-Channel Flows. Balkema.

Pan, J. \& BanerJee, S. 1995 A numerical study of free-surface turbulence in channel flow. Phys. Fluids 7, 1649-1664. 
Perkins, H. J. 1970 The formation of streamwise vorticity in turbulent flow. J. Fluid Mech. 44, $721-740$.

Pope, S. B. 2000 Turbulent Flows. Cambridge University Press.

Pourquí, M. J. B. M. 1994 Large-eddy simulation of a turbulent jet. PhD thesis, Delft University of Technology, Delft.

RozovskiI, I. L. 1957 Flow of water in bends of open channels. Acad. Sci. Ukraine SSR (Israeli Prog. Sci. Transl. 1961).

Schwarz, W. R. \& Bradshaw, P. 1994 Turbulence structural changes for a three-dimensional turbulent boundary layer in a $30^{\circ}$ bend. J. Fluid Mech. 272, 183-209.

Shi, J., Thomas, T. G. \& Williams, J. J. R. 2000 Free-surface effects in open channel flow at moderate Froude and Reynolds numbers. J. Hydr. Res. 38, 465-474.

Shiono, K. \& Muto, Y. 1998 Complex flow mechanisms in compound meandering channels with overbank flow. J. Fluid. Mech. 376, 221-261.

Simonsen, A. J. \& Krogstad, P. Å. 2005 Turbulent stress invariant analysis: clarification of existing terminology. Phys. Fluids 18, 088103.

Speziale, C. G. 1987 On nonlinear $k-l$ and $k-\epsilon$ models of turbulence. J. Fluid Mech. 178, 459-475.

Stoesser, T., Ruether, N. \& Olsen, N. R. B. 2008 Near-bed flow behaviour in a meandering channel. In Proc. Riverflow 2008, pp. 793-799. Kubaba.

TANNER, W. 1963 Spiral flow in rivers, shallow seas, dust devils, and models. Science 139, 41-42.

Tessicini, F., Iaccarino, G., Fatica, M., Wang, M. \& Verzicco, R. 2002 Wall modelling for largeeddy simulation using an immersed boundary method. CTR Ann. Res. Briefs, pp. 181-187.

Thorne, C. R., Zevenbergen, L. W., Pitlick, J. C., Rais, S., Bradley, J. B. \& Julien, P. Y. 1985 Direct measurements of secondary currents in a meandering sand-bed river. Nature 315, $746-747$.

Tominaga, A., NagaO, M. \& NezU, I. 1999 Flow structure and momentum transport processes in curved open-channels with vegetation. In Proc. 28th IAHR Congress, Graz, Austria. Institute for Hydraulics and Hydrology.

VAN DER VORST, H. A. 1992 Bi-cgstab: a fast and smoothly converging variant of bi-cg for the solution of nonsymmetric linear systems. SIAM J. Sci. Statist. Comput. 13, 631-644.

YANG, S. Q. 2005 Interactions of boundary shear stress, secondary currents and velocity. Fluid Dyn. Res. 36, 121-136.

Ye, J. \& McCorquodale, J. A. 1998 Simulation of curved open-channel flows by 3 d hydrodynamic model. J. Hydr. Engng 124, 687-698.

Zeng, J., Constantinescu, G., Blanckaert, K. \& Weber, L. 2008 Flow and bathymetry in sharp open-channel bends: experiments and predictions. Water Resour. Res. 44, W09401. 